\title{
DIFFERENTIAL OPERATORS AND CHEREDNIK ALGEBRAS
}

\author{
V. GINZBURG, I. GORDON, AND J. T. STAFFORD
}

\begin{abstract}
We establish a link between two geometric approaches to the representation theory of rational Cherednik algebras of type $\mathbf{A}$ : one based on a noncommutative Proj construction GS1; the other involving quantum hamiltonian reduction of an algebra of differential operators [GG].

In the present paper, we combine these two points of view by showing that the process of hamiltonian reduction intertwines a naturally defined geometric twist functor on $\mathscr{D}$-modules with the shift functor for the Cherednik algebra. That enables us to give a direct and relatively short proof of the key result [GS1, Theorem 1.4] without recourse to Haiman's deep results on the $n$ ! theorem [Ha1]. We also show that the characteristic cycles defined independently in these two approaches are equal, thereby confirming a conjecture from GG.
\end{abstract}

\section{Contents}

1. Introduction

2. Notation and hamiltonian reduction

3. Uniqueness of reflexive bimodules

4. Relating hamiltonian reduction and shift functors

5. Proof of Theorems 1.6 and 1.7

6. Shift functors for $\mathscr{D}$-modules and Cherednik algebras

7. Characteristic cycles

8. Appendix: The radial parts map

References

\section{INTRODUCTION}

1.1. Throughout, $W=S_{n}$ will denote the $n^{\text {th }}$ symmetric group for some $n \geq 2$. For a parameter $c \in \mathbb{C}$ we write $\mathrm{H}_{c}$ for the Cherednik algebra of type $W$ with spherical

1991 Mathematics Subject Classification. 14C05, 32S45,16S80, 16D90, 05E10.

Key words and phrases. Cherednik algebra, Hilbert scheme, characteristic varieties.

The first author was supported in part by the NSF through the grant DMS-0601050. The second author was supported by the Leverhulme Trust. The third author is a Royal Society-Wolfson Research Merit Award holder and was supported in part by the NSF through the grant DMS-0555750 and by the Leverhulme Trust. 
subalgebra $\mathrm{U}_{c}=e \mathrm{H}_{c} e$, where $e=\frac{1}{|W|} \sum_{w \in W} w \in \mathrm{H}_{c}$ is the trivial idempotent. (The formal definitions of this and related concepts can mostly be found in Section 2,)

Cherednik algebras have been influential in a wide range of subjects, having been used for instance to answer questions in real algebraic geometry, integrable systems, combinatorics, and symplectic quotient singularities. They are also closely related to the Hilbert scheme Hilb ${ }^{n} \mathbb{C}^{2}$ of points in the plane, a connection that was formalized in GS1] and $\left[\mathrm{KR}\right.$, where it was shown that one can describe $\mathrm{U}_{c}$ as a kind of noncommutative deformation of Hilb ${ }^{n} \mathbb{C}^{2}$. This was then used in [GS1, GS2] to apply the geometry of Hilb $^{n} \mathbb{C}^{2}$ and Haiman's work on the $n$ ! conjecture [Ha1] to analyse the representation theory of $\mathrm{H}_{c}$. In the process one finds that important classes of representations of the Cherednik algebra correspond to important classes of sheaves on the Hilbert scheme and, through them, to important combinatorial objects.

1.2. There are two main aims in this paper. First, the proof of the main result from GS1, Theorem 1.4, is heavily dependent on [Ha1] and so one would like to obtain a proof of that result that is independent of Haiman's work, not least because one may then be able to apply Cherednik algebra techniques to the study of $\operatorname{Hilb}^{n} \mathbb{C}^{2}$ and related combinatorial objects. Second, there are two different ways to relate representations of the Cherednik algebra $\mathrm{H}_{c}$ to geometric constructions and in particular to the Hilbert scheme: through the noncommutative geometry approach of [GS1 mentioned above; and through quantum hamiltonian reduction of the $G L_{n}(\mathbb{C})$-equivariant space $\mathfrak{g l}(\mathbb{C}) \times \mathbb{C}^{n}$, as in [GG]. One would like to understand the relationship between the two approaches.

In this paper we solve both these problems by using quantum hamiltonian reduction to give an alternative and shorter proof of [GS1, Theorem 1.4] without recourse to [Ha1]. This also clarifies the relationship between the two approaches, for example showing that the characteristic cycles defined independently in GS2] and GG] are actually equal, thereby confirming a conjecture of [GG].

Before we describe these results in detail we need to introduce some notation.

1.3. Let $\mathfrak{h}=\mathbb{C}^{n}$ denote the permutation representation of $W$ and write $\mathfrak{h}^{\text {reg }}=\mathfrak{h} \backslash \delta^{-1}(0)$ where $\delta=\prod_{i<j}\left(x_{i}-x_{j}\right) \in \mathbb{C}[\mathfrak{h}]$ is the discriminant; equivalently $\mathfrak{h}^{\text {reg }}$ is the subset of $\mathfrak{h}$ on which $W$ acts freely. The algebra $\cup_{c}$ will be identified through the Dunkl embedding with a subalgebra of $\mathscr{D}\left(\mathfrak{h}^{\text {reg }}\right) * W$, the skew group ring of $W$ with coefficients in the ring of differential operators on $\mathfrak{h}^{\text {reg }}$.

Set

$$
\mathcal{C}=\left\{c \in \mathbb{C}: c=\frac{a}{b} \text { where } a, b \in \mathbb{Z} \text { with } 2 \leq b \leq n\right\} .
$$

A scalar $c \in \mathbb{C}$ is called good provided that $c \notin \mathcal{C} \cap(-1,0)$. The point of the definition is that the good values of $c \in \mathbb{C}$ are the ones for which [GS1] and [BE] show that $\mathrm{U}_{c}$ has pleasant properties (see also Remark 2.4). 
1.4. For $a \in \mathbb{C}$, set

$$
{ }_{a} \mathrm{P}_{a-1}=e \mathrm{H}_{a} \delta e \quad \text { and } \quad{ }_{a-1} \mathrm{Q}_{a}=e \delta^{-1} \mathrm{H}_{a} e .
$$

By induction, for $a \in b+\mathbb{Z}_{\geq 2}$, define

$$
{ }_{a} \mathrm{P}_{b}=\left({ }_{a} \mathrm{P}_{a-1}\right) \cdot\left({ }_{a-1} \mathrm{P}_{b}\right) \quad \text { and } \quad{ }_{b} \mathrm{Q}_{a}=\left({ }_{b} \mathrm{Q}_{b+1}\right) \cdot\left({ }_{b+1} \mathrm{Q}_{a}\right) .
$$

In these equations, the multiplication is taken inside $\mathscr{D}\left(\mathfrak{h}^{\text {reg }}\right) * W$ and this makes both ${ }_{a} \mathrm{P}_{b}$ and ${ }_{a} \mathrm{Q}_{b}$ into $\left(\mathrm{U}_{a}, \mathrm{U}_{b}\right)$-bimodules.

The key to [GS1, GS2] is the construction of a $\mathbb{Z}$-algebra $B=\bigoplus_{i \geq j \geq 0}\left(c_{c+i} \mathrm{P}_{c+j}\right)$ endowed with a natural matrix multiplication. The ring $B$ has a natural filtration induced from the differential operator filtration $\Gamma$ on $\mathscr{D}\left(\mathfrak{h}^{\text {reg }}\right) * W$ and the main result [GS1, Theorem 1.4] showed that if $c+i$ is good for all $i \in \mathbb{N}$, then the associated graded ring $\operatorname{gr}_{\Gamma} B$ of $B$ is the $\mathbb{Z}$-algebra associated to $\operatorname{Hilb}^{n} \mathbb{C}^{2}$. This provides the bridge between Cherednik algebras and Hilbert schemes.

1.5. There is another way of passing from $\mathrm{H}_{c}$ to a more geometric setting which uses the hamiltonian reduction from [GG]. This works as follows. Write $V=\mathbb{C}^{n}$ and set $G=G L(V)$ with Lie algebra $\mathfrak{g}=\mathfrak{g l}(V)$. If $\mathfrak{G}=\mathfrak{g} \times V$ with its natural $G$-action, then GG] shows that $\mathrm{U}_{c} \cong\left(\mathscr{D}(\mathfrak{G}) / \mathscr{D}(\mathfrak{G}) \cdot I_{c+1}\right)^{G}$ for an appropriate ideal $I_{c+1}$ of $U(\mathfrak{g})$ (see Section 2.6 for the details). One of the main results of this paper shows that there is a natural interpretation of the ${ }_{a} \mathrm{Q}_{b}$ in terms of this hamiltonian reduction. Indeed, set $\mathfrak{D}_{c+1}=\mathscr{D}(\mathfrak{G}) / \mathscr{D}(\mathfrak{G}) \cdot I_{c+1}$ and, for $m \in \mathbb{Z}$, consider the space of semi-invariants

$$
\mathfrak{D}_{c+1}^{\operatorname{det}^{-m}}=\left\{D \in \mathfrak{D}_{c+1}: g \cdot D=\operatorname{det}(g)^{-m} D\right\} .
$$

1.6. It is easy to check that $\mathfrak{D}_{c+1}^{\mathrm{det}^{-m}}$ is a $\left(\mathrm{U}_{c-m}, \mathrm{U}_{c}\right)$-bimodule and our first main result shows that it is a familiar object:

Theorem. Fix $c \in \mathbb{C}$ and an integer $m \geq 1$ so that each of $c-1, c-2, \ldots, c-m+1$ is good (this is automatic if $m=1$ ). Under the differential operator filtration on the two sides there is a filtered $\left(\mathrm{U}_{c-m}, \mathrm{U}_{c}\right)$-bimodule isomorphism

$$
\Theta_{c, m}: \mathfrak{D}_{c+1}^{\operatorname{det}^{-m}} \stackrel{\sim}{\longrightarrow}{ }_{c-m} \mathbf{Q}_{c} .
$$

This also leads to a description of ${ }_{c} \mathrm{P}_{c-m}$ in terms of the $\mathfrak{D}_{d+1}^{\mathrm{det}^{-m}}$ (see Lemma 5.6).

The idea behind the proof of this theorem is easy to describe. One first shows that, like ${ }_{c-m} \mathbf{Q}_{c}$, the $\left(\mathbf{U}_{c-m}, \mathbf{U}_{c}\right)$-bimodule $\mathfrak{D}_{c+1}^{\mathrm{det}^{-m}}$ is naturally embedded into $\mathbf{U}^{\text {reg }}=\mathbf{U}_{c}\left[\delta^{-2}\right]$. Moreover, both of these bimodules are reflexive on at least one side (see Corollary 3.7 and Lemma 4.4). The theorem is then proved by showing that such a bimodule is unique (see Theorem 3.5). 
1.7. Set $A^{1}=\mathbb{C}\left[\mathfrak{h} \times \mathfrak{h}^{*}\right]^{\text {sign }}$, with powers $A^{m}$ under multiplication in $\mathbb{C}\left[\mathfrak{h} \times \mathfrak{h}^{*}\right]$. Using Theorem 1.6 we are also able to to give a direct and relatively short proof of the following result, which is essentially [GS1, Theorem 1.4] and is one of the central results from that paper.

Theorem. Keep the hypotheses of Theorem 1.6; thus $c-1, \ldots, c-m+1$, are all good.

(1) Under the differential operator filtration $\Gamma$, one has $\operatorname{gr}_{\Gamma}\left({ }_{c-m} \mathrm{Q}_{c}\right)=\delta^{-m} A^{m}$ e inside $\operatorname{gr}_{\Gamma} \mathrm{U}^{\text {reg }}=\mathbb{C}\left[\mathfrak{h}^{\text {reg }} \times \mathfrak{h}\right] e$.

(2) Similarly, $\operatorname{gr}_{\Gamma}\left({ }_{c} \mathrm{P}_{c-m}\right)=A^{m} \delta^{m} e$.

Crucially, and unlike the original proof of [GS1, Theorem 1.4], the proof of Theorem 1.7 does not depend upon Haiman's work [Ha1].

1.8. As was remarked earlier, passing from finitely generated filtered $\mathbf{H}_{c}$-modules to coherent sheaves on Hilb ${ }^{n} \mathbb{C}^{2}$ via the $\mathbb{Z}$-algebra $B=\bigoplus_{m \geq 0}{ }_{c+m} \mathrm{P}_{c}$ provides a powerful technique for studying the representation theory of $\mathrm{U}_{c}$ and $\mathrm{H}_{c}$, see GS1, GS2. Under this relation, GS1, Corollary 1.9] shows that the regular representation $\mathrm{H}_{c}$ corresponds to the Procesi bundle $\mathcal{P}$ : this is a vector bundle of rank $n$ ! over Hilb ${ }^{n} \mathbb{C}^{2}$ whose fibres carry the regular representation of $S_{n}$, see [Ha1]. These fibres describe Macdonald polynomials and have deep connections with many areas of representation theory and algebraic combinatorics.

One may hope to use Theorem 1.7 and the representation theory of $\mathrm{H}_{c}$ to provide a new construction of $\mathcal{P}$ which will explain many of its properties. This goal is still out of reach. Furthermore, the more detailed relationship between the category $\mathbf{H}_{c}$-mod of finitely generated left $\mathrm{H}_{c}$-modules and the category $\mathrm{Coh}\left(\mathrm{Hilb}^{n} \mathbb{C}^{2}\right)$ of coherent sheaves on Hilb ${ }^{n} \mathbb{C}^{2}$ established in GS2 is itself dependent upon several important properties of the construction of $\mathcal{P}$ in Ha1. So there is much to understand.

1.9. In the second part of the paper we use Theorems 1.6 and 1.7 to relate other structures appearing in the $\mathbb{Z}$-algebra and quantum hamiltonian reduction constructions. These are concerned with the functor of hamiltonian reduction

$$
\widetilde{\mathbb{H}}_{c}:\left(\mathscr{D}_{-c}(\mathfrak{X}), S L(V)\right)-\bmod \longrightarrow \mathrm{U}_{c^{-}} \bmod
$$

where $\mathfrak{X}=\mathfrak{g} \times \mathbb{P}^{n-1}$ and $\left(\mathscr{D}_{-c}(\mathfrak{X}), S L(V)\right)$-mod denotes the category of finitely generated $S L(V)$-equivariant $\mathscr{D}$-modules on $\mathfrak{X}$ that are $n c$-twisted on $\mathbb{P}^{n-1}$. The functor $\widetilde{\mathbb{H}}_{c}$ is defined formally in (6.6) but, up to a shift, is given by $\mathcal{F} \longmapsto \mathcal{F}^{S L(V)}$. This functor has been used for example in [FG] to relate representations of $U_{c}$ with generalisations of Lusztig's character sheaves.

Working with $\mathscr{D}(\mathfrak{X})$ rather than $\mathscr{D}(\mathfrak{G})$ is not particularly significant since one can pass from the latter to the former by taking invariants under the central subgroup $\mathbb{C}^{\times} \subset G L(V)$. What is significant is that there is now a natural translation functor $\mathbb{S}^{m}:\left(\mathscr{D}_{-c}(\mathfrak{X}), S L(V)\right)$-mod $\rightarrow\left(\mathscr{D}_{-c-m}(\mathfrak{X}), S L(V)\right)$-mod given by tensoring with 
the sheaf $\mathcal{O}_{\mathbb{P}(V)}(n m)$ on $\mathbb{P}(V)$. On the other hand, one has the translation functor ${ }_{c+m} \mathrm{P}_{c} \otimes(-): \mathrm{U}_{c}$-mod $\rightarrow \mathrm{U}_{c+m}$-mod; when $c, c+1, \cdots, c+m$ are good this is an equivalence of categories that plays a crucial rôle in [GS1, GS2], analogous to the translation principle in Lie theory. As we prove, it also has a natural description in terms of $\widetilde{\mathbb{H}}_{c}$ and its left adjoint ${ }^{\top} \widetilde{\mathbb{H}}_{c}$.

Theorem. Assume that $n>2$ and that $c \in \mathbb{C} \backslash \mathbb{Q}<0$. Then, for all integers $m \geq 0$, there is an isomorphism of functors $\widetilde{\mathbb{H}}_{c+m} \circ \mathbb{S}^{m} \circ{ }^{\top} \widetilde{\mathbb{H}}_{c}(-) \cong{ }_{c+m} \mathrm{P}_{c} \otimes \mathrm{U}_{c}(-)$ that makes the following diagram commute

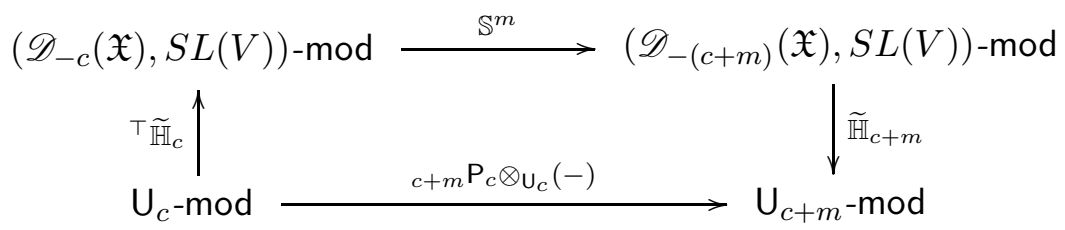

1.10. A useful tool in the study of Cherednik algebras, just as for Lie algebras, is the concept of the characteristic cycle of a $\mathrm{U}_{c}$-module (this is the same as the characteristic variety, except that it counts multiplicities of the irreducible components). In fact there are two completely different constructions of characteristic cycles of $\mathrm{U}_{c^{-}}$-modules on Hilb $^{n} \mathbb{C}^{2}$ : the first, $\mathrm{ch}^{G S}$, uses the $\mathbb{Z}$-algebra approach form [GS1]; the second, $\mathrm{ch}^{G G}$, is defined using the machinery of hamiltonian reduction. In our final result we prove that these two constructions agree, thereby confirming a conjecture from [GG, (7.17)].

Theorem. Assume that $n>2$ and that $c \in \mathbb{C} \backslash \mathbb{Q}<0$. Then for any finitely generated $\mathrm{U}_{c}$-module $M$ one has an equality of algebraic cycles $\mathrm{ch}^{G S}(M)=\mathrm{ch}^{G G}(M)$.

1.11. The paper is organised as follows. In Section 2 we introduce the basic notation and background material. Section 3 is the key to the paper: it gives a uniqueness result for reflexive $\left(\mathrm{U}_{c+m}, \mathrm{U}_{c}\right)$-bisubmodules of $\mathscr{D}\left(\mathfrak{h}^{\text {reg }}\right) * W$. We use this in Section 4 to prove Theorem 1.6 in the special case when $m=1$, while in Section 5 we extend this to prove Theorems 1.6 and 1.7 in general. In Sections 6 and 7 we prove slightly stronger versions of Theorems 1.9 and 1.10, respectively, which also include the case $n=2$. Finally in the appendix we give a detailed proof of the version of hamiltonian reduction that we need, since it does not follow directly from that in [GG].

\section{Notation AND HAMiltonian REDUCtion}

2.1. Differential operators. Let $G$ be a reductive algebraic group with Lie algebra $\mathfrak{g}=\operatorname{Lie}(G)$ and write $U(\mathfrak{g})$ for the enveloping algebra of $\mathfrak{g}$. Let $X$ be a smooth affine algebraic variety with coordinate ring $\mathbb{C}[X]$ and ring of regular algebraic differential operators $\mathscr{D}(X)$. Assume that $G$ acts algebraically on $X$. This gives rise to a locallyfinite $G$-action on $\mathbb{C}[X]$ and $\mathscr{D}(X)$ via the formulæ:

$$
(g \cdot f)(x) \stackrel{\text { def }}{=} f\left(g^{-1} \cdot x\right) \quad \text { and } \quad(g \cdot \theta)(f) \stackrel{\text { def }}{=} g \cdot\left(\theta\left(g^{-1} \cdot f\right)\right),
$$


for $g \in G, f \in \mathbb{C}[X], \theta \in \mathscr{D}(X)$ and $x \in X$.

We should emphasise that this is not the action used in [GG] and [BFG]; those papers implicitly use the rule $(g \cdot f)(x)=f(g \cdot x)$ for $g \in G, f \in \mathbb{C}[X]$ and $x \in X$. (See [GG, Equation A4] and the comments after [BFG, Lemma 5.3.3].)

The action of $G$ on $\mathbb{C}[X]$ and $\mathscr{D}(X)$ is by given algebra automorphisms, and we let $\mathscr{D}(X)^{G} \subset \mathscr{D}(X)$ denote the subalgebra of $G$-invariant differential operators. The action of $G$ on $X$ gives rise to a $\mathfrak{g}$-action on $\mathbb{C}[X]$ by derivations and this induces a Lie algebra map $\tau: \mathfrak{g} \rightarrow \operatorname{Der}(\mathbb{C}[X]) \subset \mathscr{D}(X)$. This extends uniquely to an associative algebra morphism $\tau: U(\mathfrak{g}) \rightarrow \mathscr{D}(X)$.

2.2. Given a group character $\chi: G \rightarrow \mathbb{C}^{\times}$and a $G$-module $M$, write

$$
M^{\chi}=\{m \in M \quad \mid g \cdot m=\chi(g) m, \forall g \in G\}
$$

for the corresponding $\chi$-isotypic component. Abusing notation, we also write $\chi: \mathfrak{g} \rightarrow \mathbb{C}$ for the differential of the group character $\chi$.

Let $\nu: \mathfrak{g} \rightarrow \mathbb{C}$ be a Lie algebra character and write $I_{\nu}$ for the two-sided ideal in $U(\mathfrak{g})$ generated by the elements $\{x-\nu(x): x \in \mathfrak{g}\}$. It is standard that multiplication in $\mathscr{D}(X)$ induces an algebra structure on the space of $G$-invariants $\left[\mathscr{D}(X) / \mathscr{D}(X) \tau\left(I_{\nu}\right)\right]^{G},[\mathrm{BFG}$, Section 3.4], called the quantum hamiltonian reduction of $\mathscr{D}(X)$ at $\nu$. Similarly, for a character $\chi$ of $G$, the next lemma shows that we obtain a natural bimodule structure for the isotypic component $\left[\mathscr{D}(X) / \mathscr{D}(X) \tau\left(I_{\nu}\right)\right]^{\chi}$. For notational simplicity we will often write $\mathscr{D}(X) I_{\nu}$ in place of $\mathscr{D}(X) \tau\left(I_{\nu}\right)$.

Lemma. Let $\chi: G \longrightarrow \mathbb{C}^{\times}$be a group character and $\nu: \mathfrak{g} \longrightarrow \mathbb{C}$ a Lie algebra character. Multiplication in the algebra $\mathscr{D}(X)$ endows $\left[\mathscr{D}(X) / \mathscr{D}(X) \tau\left(I_{\nu}\right)\right]^{\chi}$ with the structure of a right $\left[\mathscr{D}(X) / \mathscr{D}(X) \tau\left(I_{\nu}\right)\right]^{G}$-module and a left $\left[\mathscr{D}(X) / \mathscr{D}(X) \tau\left(I_{\nu+\chi}\right)\right]^{G}$-module.

Proof. The right $\left[\mathscr{D}(X) / \mathscr{D}(X) \tau\left(I_{\nu}\right)\right]^{G}$-module structure is obvious. The differential of the $G$-action on $\mathscr{D}(X)$ induces an action of $\tau(\mathfrak{g})$ by commutation: thus if $x \in \mathfrak{g}$ and $D \in \mathscr{D}(X)^{\chi}$ then $[\tau(x), D]=\chi(x) D$. Hence

$$
(\tau(x)-(\nu+\chi)(x)) D=D(\tau(x)-\nu(x)) \in \mathscr{D}(X) \tau\left(I_{\nu}\right)
$$

and so the left action of $\mathscr{D}(X)^{G}$ on $\left[\mathscr{D}(X) / \mathscr{D}(X) \tau\left(I_{\nu}\right)\right]^{\chi}$ factors through the factor ring $\left[\mathscr{D}(X) / \mathscr{D}(X) \tau\left(I_{\nu+\chi}\right)\right]^{G}$.

2.3. Rational Cherednik algebras. Fix a positive integer $n \geq 2$, let $W=S_{n}$ be the symmetric group and write $e=\frac{1}{|W|} \sum_{w \in W} w \in \mathbb{C} W$ for the trivial idempotent. Similarly, let $e_{-}=\frac{1}{|W|} \sum_{w \in W} \operatorname{sign}(w) \cdot w$ denote the sign idempotent. Recall that $\mathfrak{h}=\mathbb{C}^{n}$ is the permutation representation of $W$ and that

$$
\mathfrak{h}^{\text {reg }}=\left\{\left(z_{1}, \ldots, z_{n}\right) \in \mathfrak{h}: z_{i} \neq z_{j} \text { for all } 1 \leq i<j \leq n\right\}
$$


denotes the subset of $\mathfrak{h}$ on which $W$ acts freely. Equivalently, if $\left\{x_{1}, \ldots, x_{n}\right\}$ is the basis of $\mathfrak{h}^{*} \subset \mathbb{C}[\mathfrak{h}]$ consisting of coordinate functions, then $\mathfrak{h}^{\text {reg }}=\mathfrak{h} \backslash \delta^{-1}(0)$ where $\delta=\prod_{i<j}\left(x_{i}-x_{j}\right) \in \mathbb{C}[\mathfrak{h}]$ is the discriminant. There is an induced action of $W$ on $\mathscr{D}\left(\mathfrak{h}^{\text {reg }}\right)$ and we write $\mathscr{D}\left(\mathfrak{h}^{\text {reg }}\right) * W$ for the corresponding skew group ring; for $D \in \mathscr{D}\left(\mathfrak{h}^{\text {reg }}\right)$ and $w \in W$ multiplication is defined by $w D=(w \cdot D) w$.

Fix a scalar $c \in \mathbb{C}$ and let $\mathrm{H}_{c}$ denote the rational Cherednik algebra corresponding to $G L_{n}(\mathbb{C})$. As in [EG, Proposition 4.5], we will identify $\mathrm{H}_{c}$ with the subalgebra of $\mathscr{D}\left(\mathfrak{h}^{\text {reg }}\right) * W$ generated by $W$, the vector space $\mathfrak{h}^{*}=\sum x_{i} \mathbb{C} \subset \mathbb{C}[\mathfrak{h}]$ of linear functions, and the Dunkl operators

$$
D_{c}\left(y_{i}\right)=\frac{\partial}{\partial x_{i}}-\frac{1}{2} \sum_{j \neq k} c \frac{\left\langle y_{i}, x_{j}-x_{k}\right\rangle}{x_{j}-x_{k}}\left(1-s_{j k}\right)
$$

where $\left\{y_{1}, \ldots, y_{n}\right\} \subset \mathfrak{h}$ is the dual basis to $\left\{x_{1}, \ldots, x_{n}\right\}$ and the $s_{j k} \in W$ are simple transpositions. By [EG, Theorem 1.3] there is a PBW isomorphism $\mathrm{H}_{c} \cong \mathbb{C}[\mathfrak{h}] \otimes \mathbb{C} W \otimes \mathbb{C}\left[\mathfrak{h}^{*}\right]$ of $\mathbb{C}$-vector spaces. The spherical subalgebra of $\mathrm{H}_{c}$ is $\mathrm{U}_{c}=e \mathrm{H}_{c} e$.

Clearly $\delta^{2} \in \mathbb{C}[\mathfrak{h}]^{W}$. Let $\mathrm{U}^{\text {reg }}=\mathrm{U}_{c}\left[\delta^{-2}\right]$ and $\mathrm{H}^{\text {reg }}=\mathrm{H}_{c}\left[\delta^{-2}\right]=\mathrm{H}_{c}\left[\delta^{-1}\right]$ be the corresponding localised algebras. By definition, $\mathscr{D}\left(\mathfrak{h}^{\text {reg }}\right)=\mathscr{D}(\mathfrak{h})\left[\delta^{-1}\right]$ and, by $[$ EG, Proposition 4.5], $\mathrm{U}^{\text {reg }}=e \mathscr{D}\left(\mathfrak{h}^{\text {reg }}\right)^{W} e=e\left(\mathscr{D}\left(\mathfrak{h}^{\text {reg }}\right) * W\right) e$ is independent of the choice of $c$.

2.4. Remark. (i) If $\mathrm{H}_{c}^{\prime}$ is the corresponding $S L_{n}(\mathbb{C})$ version of the Cherednik algebra with spherical subalgebra $\mathrm{U}_{c}^{\prime}$ then $\mathrm{H}_{c} \cong \mathrm{H}_{c}^{\prime} \otimes_{\mathbb{C}} \mathscr{D}\left(\mathbb{A}^{1}\right)$ and $\mathrm{U}_{c} \cong \mathrm{U}_{c}^{\prime} \otimes \mathscr{D}\left(\mathbb{A}^{1}\right)$. It is therefore straightforward to apply the results of (for example) [GS1] to $\mathrm{H}_{c}$ and $\mathrm{U}_{c}$.

(ii) The results in GS1 also assumed that $c \notin \frac{1}{2}+\mathbb{Z}$, but this condition has since been removed by $[\mathrm{BE}]$. So all the results in [GS1, GS2] can now be applied without that hypothesis. Thus, for example, GS1, Corollary 3.13] and [BE, Theorem 4.1] show that if $c \in \mathbb{C}$ is good, then $\mathrm{U}_{c}$ is Morita equivalent to $\mathrm{H}_{c}$ and consequently has finite homological global dimension.

2.5. The order of differential operators induces a filtration on $\mathscr{D}\left(\mathfrak{h}^{\text {reg }}\right) * W$ by putting $W$ into degree zero. Essentially every (noncommutative) ring $R$ or module $M$ that we consider is naturally embedded as a subfactor of either $\mathscr{D}\left(\mathfrak{h}^{\text {reg }}\right) * W$ or $\mathscr{D}(X)$ for some variety $X$ : we will call the induced filtration on $R$ and $M$ the differential operator filtration and this will usually be written as $M=\bigcup_{j \geq 0} \Gamma_{i} M$. The PBW isomorphism can then be rephrased as saying that there are algebra isomorphisms $\operatorname{gr}_{\Gamma} \mathrm{H}_{c} \cong \mathbb{C}\left[\mathfrak{h} \times \mathfrak{h}^{*}\right] * W$ and $\operatorname{gr}_{\Gamma} \mathrm{U}_{c} \cong \mathbb{C}\left[\mathfrak{h} \times \mathfrak{h}^{*}\right]^{W}$.

2.6. Quantum hamiltonian reduction. Fix an $n$-dimensional $\mathbb{C}$-vector space $V$ and put $G=G L(V)$ with Lie algebra $\mathfrak{g}=\mathfrak{g l}(V)=\operatorname{Lie}(G) \supset \mathfrak{s l}(V)$. Write $\mathfrak{G}=\mathfrak{g} \times V$ with the $G$-action $g \cdot(X, v)=\left(g X g^{-1}, g v\right)$ for $g \in G$ and $(X, v) \in \mathfrak{g} \times V$. Let $\mathbf{1} \in \mathfrak{g}$ denote the identity. For any $c \in \mathbb{C}$, let $\chi_{c}: \mathfrak{g} \rightarrow \mathbb{C}$ be the Lie algebra homomorphism defined by 
$x \mapsto c \cdot \operatorname{Tr}(x)$. For simplicity we write $I_{c}=I_{\chi_{c}} \subset U(\mathfrak{g})$ : thus $I_{c}=U(\mathfrak{g}) \mathfrak{s l}_{n}+U(\mathfrak{g})(\mathbf{1}-n c)$. Much of the paper will be concerned with the objects

$$
\mathfrak{D}_{c} \stackrel{\text { def }}{=} \frac{\mathscr{D}(\mathfrak{G})}{\mathscr{D}(\mathfrak{G}) \tau\left(I_{c}\right)} \quad \text { and } \quad \mathfrak{D}_{c}^{G} \stackrel{\text { def }}{=}\left[\frac{\mathscr{D}(\mathfrak{G})}{\mathscr{D}(\mathfrak{G}) \tau\left(I_{c}\right)}\right]^{G},
$$

which, following the earlier convention, are given the differential operator filtration $\Gamma$ induced from that on $\mathscr{D}(\mathfrak{G})$.

2.7. The action of $\mathbf{1}$ on $\mathbb{C}[\mathfrak{G}]$ will be used a number of times in this paper and so it is appropriate to be explicit about it. The action of the centre $\mathbb{C}^{\times}$of $G$ on $\mathfrak{G}$ is given by dilation in the second component: $\lambda \cdot(X, v)=(X, \lambda v)$ for $\lambda \in \mathbb{C}^{\times}$and $(X, v) \in \mathfrak{g} \times V=\mathfrak{G}$. By (2.1.1), $\mathbb{C}^{\times}$therefore acts on $V^{*} \subset \mathbb{C}[V]$ by anti-dilation. Since the action of $\mathfrak{g}$ is the differential of the $G$-action, this implies that the action of $\tau(\mathbf{1})$ will be concentrated purely on $\mathbb{C}[V]$ and that it will then be the negative of the Euler operator. In other words, if $\left\{e_{i}\right\}$ is a basis of $V^{*} \subset \mathbb{C}[V]$ then $\tau(\mathbf{1})=-\sum_{i=1}^{n} e_{i} \frac{\partial}{\partial e_{i}} \in \mathscr{D}(V) \subset \mathscr{D}(\mathfrak{G})$.

2.8. One of the main results of [GG] shows that $\mathrm{U}_{c}$ may be obtained from $\mathscr{D}(\mathfrak{G})$ via quantum hamiltonian reduction.

Theorem. With the differential operator filtrations described above, there is for every $c \in \mathbb{C}$ an isomorphism of filtered algebras $\mathfrak{D}_{c}^{G} \cong \mathrm{U}_{c-1}$. This induces an isomorphism of graded algebras $\operatorname{gr} \mathfrak{D}_{c}^{G} \cong \operatorname{gr} \mathbf{U}_{c-1} \cong \mathbb{C}\left[\mathfrak{h} \times \mathfrak{h}^{*}\right]^{W}$.

Proof. This result is a variant of [GG, Theorem 1.5], but since the result does not follow directly from the results in that paper, we give a complete proof in the appendix.

\section{UNIQUENESS OF REFLEXIVE BIMODULES}

3.1. The way we will prove the isomorphism $\mathfrak{D}_{c+1}^{\text {det }^{-m}} \stackrel{\sim}{\longrightarrow}{ }_{c-m} \mathbf{Q}_{c}$ of Theorem [1.6 is to note that both sides are isomorphic to $\left(\mathrm{U}_{c-m}, \mathbf{U}_{c}\right)$-bisubmodules of $\mathscr{D}\left(\mathfrak{h}^{\text {reg }}\right) * W$ that are reflexive $\mathrm{U}_{c-m}$-modules. The isomorphism will then follow once we know that such a bimodule is unique. The aim of this section is to prove such a uniqueness result, but since the idea works for more than just spherical algebras we will prove it under the following general hypotheses. In what follows we write $\operatorname{GKdim}(M)$ for the GelfandKirillov dimension of a module $M$.

3.2. Hypotheses. We assume that $(S, \Gamma)$ is a filtered algebra over a field $k$ such that $\operatorname{gr}_{\Gamma} S$ is a commutative domain. Assume that $R_{1}$ and $R_{2}$ are two subalgebras of $S$ such that:

(1) Each $R_{i}$ is a Goldie domain with $S$ contained in the common Goldie quotient ring $F$ of the $R_{i}$.

(2) Under the induced filtration $\Gamma$, the rings $\operatorname{gr}_{\Gamma} R_{i}$ are Gorenstein algebras that are finitely generated modules over a common graded finitely generated $k$-algebra $C$. 
(3) For each nonzero ideal $I$ of $R_{i}$ we have GKdim $R_{i} / I \leq \operatorname{GKdim} R_{i}-2$.

3.3. We first check that Hypotheses 3.2 are satisfied by $\mathrm{U}_{c}$.

Lemma. For $i=1,2$, let $R_{i}=a_{i} \cup_{d_{i}} a_{i}^{-1} \subseteq S=\mathrm{U}^{\mathrm{reg}}$, for some $d_{i} \in \mathbb{C}$ and nonzero $a_{i} \in \mathrm{U}^{\mathrm{reg}}$. Filter $S$ and its subsets by the differential operator filtration. Then Hypotheses 3.2 are satisfied by $R_{1}$ ad $R_{2}$.

Proof. By [EG, Theorem 1.3] and [GS1, Lemma 6.8(1)],

$$
\operatorname{gr}_{\Gamma} R_{1}=\operatorname{gr}_{\Gamma} R_{2}=\operatorname{gr}_{\Gamma} e H_{d_{1}} e=\mathbb{C}\left[\mathfrak{h} \times \mathfrak{h}^{*}\right]^{W},
$$

which is Gorenstein by Watanabe's Theorem Wa. Thus parts (1) and (2) of Hypotheses $\underline{3.2}$ follow, with $C=\mathbb{C}\left[\mathfrak{h} \times \mathfrak{h}^{*}\right]^{W}$.

In order to show that part (3) holds, it suffices to work with $R_{1}=\mathrm{U}_{c}$. Since $\mathrm{U}^{\text {reg }} \cong$ $\mathscr{D}\left(\mathfrak{h}^{\text {reg }}\right)^{W}$, it is a simple ring and so $\delta^{2 m} \in I$ for some $m>0$. On the other hand, there is a "Fourier" automorphism $\kappa$ of $\bigcup_{c}$ that maps $\mathfrak{h}$ to $\mathfrak{h}^{*}$, see [EG, p. 283]. Thus, the ideal $\kappa^{-1}(I)$ contains $\delta^{2 n}$ for some $n>0$ and so $\kappa(\delta)^{2 n} \in I \cap \mathbb{C}\left[\mathfrak{h}^{*}\right]$. The PBW isomorphism EG, Theorem 1.3] implies that $\operatorname{gr} \mathrm{U}_{c} \cong \mathbb{C}\left[\mathfrak{h} \oplus \mathfrak{h}^{*}\right]^{W}$ is finitely generated as a module over its subring $\mathbb{C}[\mathfrak{h}]^{W} \otimes_{\mathbb{C}} \mathbb{C}\left[\mathfrak{h}^{*}\right]^{W}$. Consequently, $\operatorname{gr}\left(\mathrm{U}_{c} / I\right)$ is a finitely generated as a module over the algebra $\mathbb{C}[\mathfrak{h}]^{W} /\left(\delta^{2 m}\right) \otimes_{\mathbb{C}} \mathbb{C}\left[\mathfrak{h}^{*}\right]^{W} /\left(\sigma^{2 n}\right)$, where $\sigma$ is the principal symbol of $\kappa(\delta)$. This algebra has Gelfand-Kirillov dimension at most $2 \operatorname{dim} \mathfrak{h}-2$, and hence so does $\mathrm{U}_{c} / I$.

3.4. Keep the assumptions of Hypotheses 3.2 and set $R=R_{1}$. Given a non-zero finitely generated left $R$-module $M \subset F$ then [MR, Proposition 3.1.15] shows that there is a canonical identification $\operatorname{Hom}_{R}(M, R)=\{f \in F \mid M f \subseteq R\}$. The analogous result holds for right modules and the reflexive hull of $M$ is the $R$-module $M^{* *}=$ $\operatorname{Hom}_{R}\left(\operatorname{Hom}_{R}(M, R), R\right) \subset F$. Clearly $M \subseteq M^{* *}$ and $M$ is reflexive if $M=M^{* *}$. Note that when $M={ }_{c+1} \mathrm{P}_{c}$ or ${ }_{c} \mathrm{Q}_{c+1}$ as modules over either $R=\mathrm{U}_{c}$ or $R=\mathrm{U}_{c+1}$ these identifications take place inside $S=\mathrm{U}^{\mathrm{reg}}$.

We will need the following application of a theorem of Gabber.

Proposition. Keep the assumptions of Hypotheses [3.2, set $R=R_{1}$ and let $M$ be a non-zero finitely generated left $R$-submodule of $F$. Then $M^{* *}$ is the unique largest left $R$-submodule $M^{\prime} \subset F$ containing $M$ and such that $\operatorname{GKdim} M^{\prime} / M \leq \operatorname{GKdim} R-2$.

Proof. Define the grade of a finitely generated $R$-module $N$ to be

$$
j(N)=\min \left\{k: \operatorname{Ext}_{R}^{k}(N, R) \neq 0\right\} .
$$

By Hypotheses $3.2(2)$ we may apply [Bj, Theorems 4.1 and 4.3] to conclude that $R$ is ASGorenstein and that $j(N)=\operatorname{GKdim} R-\operatorname{GKdim} N$ for all finitely generated $R$-modules $N$. Thus if $M \subseteq M^{\prime}$ are finitely generated $R$-submodules of $F$, then GKdim $M^{\prime} / M \leq$ GKdim $R-2$ if and only if $j\left(M^{\prime} / M\right) \geq 2$. Such modules $M^{\prime}$ are called tame pure 
extensions of $M$. By [BjE, Theorem 3.6], if $M^{\prime}$ is any tame pure extension of $M$ then there exists an injection $\alpha: M^{\prime} \hookrightarrow M^{* *}$ that is the identity on $M$. But since $M^{\prime} / M$ is a torsion $R$-module, for any $m^{\prime} \in M^{\prime}$ there exists non-zero $r \in R$ such that $r m^{\prime} \subseteq M$ and so $r \alpha\left(m^{\prime}\right)=\alpha\left(r m^{\prime}\right)=r m^{\prime}$. This proves that $\alpha\left(m^{\prime}\right)=m^{\prime}$ for all $m^{\prime} \in M^{\prime}$ and finishes the proof of the proposition.

3.5. We are now ready to prove our uniqueness result for reflexive bimodules.

Theorem. Assume that $\left(R_{1}, R_{2}, S\right)$ satisfy Hypotheses 3.2 and let $M$ be a non-zero $\left(R_{1}, R_{2}\right)$-bisubmodule of $S$ that is finitely generated and reflexive on one side. Then it is reflexive and finitely generated on the other side and is the unique such object.

Proof. By symmetry, we may assume that $M$ is a finitely generated reflexive left $R_{1^{-}}$ module; thus $M=M^{* *} \stackrel{\text { def }}{=} \operatorname{Hom}_{R_{1}}\left(\operatorname{Hom}_{R_{1}}\left(M, R_{1}\right), R_{1}\right)$. Part (1) of Hypotheses 3.2 implies that, as a left $R_{1}$-module, $M \subseteq R_{1} f$ for some $f \in S$ from which we conclude that $\operatorname{gr}_{\Gamma} M \subseteq\left(\operatorname{gr}_{\Gamma} R_{1}\right) \sigma(f)$, where $\sigma(f)$ is the principal symbol of $f$. By Hypotheses $3.2(2)$ $\operatorname{gr}_{\Gamma} M$ is therefore a finitely generated (left) module over both gr $R_{1}$ and $C$. But $\Gamma$ is also a filtration of $M$ as a right $R_{2}$-module. Since $\operatorname{gr}_{\Gamma} M$ is a finitely generated right $C$-module it is also finitely generated as a right $\operatorname{gr}_{\Gamma} R_{2}$-module. Thus $M$ is a finitely generated right $R_{2}$-module, see [KL, Proposition 6.4]. We remark that it now follows from [ $\mathrm{KL}$, Corollary 3.4] that the Gelfand-Kirillov dimension of $M$ as a left $R_{1}$-module equals that of $M$ as a right $R_{2}$-module and we need not distinguish between them.

Next, suppose that $M$ is not unique and that $N$ is a second such bimodule. Let $\bar{M}=(M+N) / N$; by symmetry we may assume that $\bar{M} \neq 0$. Then $\bar{M}$ is a finitely generated left $R_{1}$-module, say $\bar{M}=\sum_{j 1}^{r} R_{1} \bar{m}_{i}$. Since $R_{2}$ is an Ore domain, $\bar{M}$ is a torsion right $R_{2}$-module and so

$$
I=\mathrm{r}-\operatorname{ann}_{R_{2}} \bar{M}=\bigcap_{j=1}^{r} \mathrm{r}-\operatorname{ann}_{R_{2}} \bar{m}_{j} \neq 0 .
$$

By Hypotheses $3.2(3)$ this implies that GKdim $\bar{M} \leq \operatorname{GKdim} R_{2} / I \leq \operatorname{GKdim} R_{2}-2$. Thus we also have $\operatorname{GKdim}(M+N) / M \leq \operatorname{GKdim} R_{1}-2$ as left $R_{1}$-modules. Our hypotheses mean that Proposition 3.4 can be applied, from which it follows that $N \subseteq(M+N) \subseteq$ $M^{* *}=M$. By symmetry, $M=N$ and so $M$ is indeed unique.

It remains to prove that $M$ is reflexive as a right $R_{2}$-module, so suppose to the contrary that $N=\operatorname{Hom}_{R_{2}}\left(\operatorname{Hom}_{R_{2}}\left(M, R_{2}\right), R_{2}\right) \supsetneq M$. By Proposition 3.4, again, it follows that GKdim $N / M \leq \operatorname{GKdim} R_{2}-2$. But as $M$ is a left $R_{1}$-module, so is $N$ and so by applying Proposition 3.4 to the left $R_{1}$-modules $M \subseteq N$ we conclude that $N \subseteq M^{* *}=M$.

3.6. In applications of Theorem 3.5 it is important to be careful to ensure that the bimodule structure of $M$ is induced from that of $S$; after all, for any nonzero $s \in S$ the vector space $R_{1} s$ is an $\left(R_{1}, s^{-1} R_{1} s\right)$-bimodule and hence, up to isomorphism, an $\left(R_{1}, R_{1}\right)$-bimodule. 
We are going to apply Theorem 3.5 to the modules ${ }_{c+1} \mathrm{P}_{c}$ and ${ }_{c} \mathrm{Q}_{c+1}$ from Section 1.4 and so it is worth emphasizing that no such problems occur here. Indeed, from [BEG2, Proposition 4.1], $\mathrm{U}_{c}=e \delta^{-1} \mathrm{H}_{c+1} \delta e$ as subrings of $\mathrm{U}^{\mathrm{reg}}$. Therefore, the $\left(\mathrm{U}_{c+1}, \mathrm{U}_{c}\right)$ bimodule structure of ${ }_{c+1} \mathrm{P}_{c}=e \mathrm{H}_{c+1} \delta e$ is indeed induced from multiplication in $S=\mathrm{U}^{\mathrm{reg}}$. Similar comments apply to ${ }_{c} \mathrm{Q}_{c+1}$.

3.7. We are now ready apply Theorem 3.5 to ${ }_{c+1} \mathrm{P}_{c}$ and ${ }_{c} \mathrm{Q}_{c+1}$.

Corollary. (1) As a right $\mathrm{U}_{c}$-module, ${ }_{c+1} \mathrm{P}_{c}$ is projective whenever $c$ is good. As a left $\mathrm{U}_{c+1}$-module, ${ }_{c+1} \mathrm{P}_{c}$ is projective whenever $c+1$ is good. For all values of $c$, the module ${ }_{c+1} \mathrm{P}_{c}$ is reflexive on both sides.

(2) As a left $\mathrm{U}_{c}$-module, ${ }_{c} \mathrm{Q}_{c+1}$ is projective whenever $c$ is good. As a right $\mathrm{U}_{c+1}$ module, ${ }_{c} \mathrm{Q}_{c+1}$ is projective whenever $c+1$ is good. For all values of $c$, the module ${ }_{c} \mathrm{Q}_{c+1}$ is reflexive on both sides.

(3) For all values of $c$, and under their natural embedding into $\mathrm{U}^{\mathrm{reg}}$, we have

$$
\begin{aligned}
& { }_{c+1} \mathrm{P}_{c}=\operatorname{Hom}_{\mathrm{U}_{c}}\left({ }_{c} \mathrm{Q}_{c+1}, \mathrm{U}_{c}\right)=\operatorname{Hom}_{\mathrm{U}_{c+1}}\left({ }_{c} \mathrm{Q}_{c+1}, \mathrm{U}_{c+1}\right), \quad \text { resp. } \\
& { }_{c} \mathrm{Q}_{c+1}=\operatorname{Hom}_{\mathrm{U}_{c}}\left({ }_{c+1} \mathrm{P}_{c}, \mathrm{U}_{c}\right)=\operatorname{Hom}_{\mathrm{U}_{c+1}}\left({ }_{c+1} \mathrm{P}_{c}, \mathrm{U}_{c+1}\right) .
\end{aligned}
$$

Proof. (1,2) A slightly weaker version of this result is given in GS1, but we give a different proof since we will need the argument later. We will just prove the result for $Q={ }_{c} \mathrm{Q}_{c+1}$; the same argument works for part (1).

Under the differential operator filtration $\Gamma$, the proof of [GS1, Lemma 6.9(2)] shows that $\operatorname{gr}_{\Gamma} Q \cong A^{1}=\mathbb{C}\left[\mathfrak{h} \times \mathfrak{h}^{*}\right]^{\text {sign }}$ as modules over $A^{0}=\mathbb{C}\left[\mathfrak{h} \times \mathfrak{h}^{*}\right]^{W}$. By the HochsterEagon Theorem, $\mathbb{C}\left[\mathfrak{h} \times \mathfrak{h}^{*}\right]$ is a Cohen-Macaulay $\mathbb{C}\left[\mathfrak{h} \times \mathfrak{h}^{*}\right]^{W}$-module and hence so is its summand $\mathbb{C}\left[\mathfrak{h} \times \mathfrak{h}^{*}\right]^{\text {sign }}$. By $[\mathrm{Bj}$, Corollary 3.12] we deduce that $Q$ is a torsion-free, Cohen-Macaulay $\mathrm{U}_{c+1}$-module in the sense that $\operatorname{Ext}_{\mathbf{U}_{c+1}}^{j}\left(Q, \mathrm{U}_{c+1}\right)=0$ for $j>0$. But, if $c+1$ is good, then $\mathrm{U}_{c+1}$ has finite global dimension by [GS1, Corollary 3.15] and [BE, Corollary 4.3] and so this implies that $Q$ is a projective right $\mathrm{U}_{c+1}$-module.

The analogous argument shows that $Q$ is a projective left $\mathrm{U}_{c}$-module whenever $c$ is good and so for any value of $c$ this implies that on at least one side $Q={ }_{c} Q_{c+1}$ is projective and hence reflexive. By Theorem 3.5 and Lemma 3.3, $Q$ is then reflexive on the other side.

(3) Since ${ }_{c} \mathrm{Q}_{c+1}$ is a finitely generated $\left(\mathrm{U}_{c}, \mathrm{U}_{c+1}\right)$-bisubmodule of $\mathrm{U}^{\text {reg }}$ that is reflexive on both sides, the dual module ${ }_{c} \mathrm{Q}_{c+1}^{*}=\operatorname{Hom}_{\mathrm{U}_{c}}\left({ }_{c} \mathrm{Q}_{c+1}, \mathrm{U}_{c}\right)$ is a nonzero $\left(\mathrm{U}_{c+1}, \mathrm{U}_{c}\right)$ bisubmodule of $\mathrm{U}^{\mathrm{reg}}$ which is reflexive and finitely generated as a right $\mathrm{U}_{c}$-module. But, by part (1), the same is true of ${ }_{c+1} \mathrm{P}_{c}$. Hence ${ }_{c} \mathrm{Q}_{c+1}^{*}={ }_{c+1} \mathrm{P}_{c}$ by Theorem 3.5 and Lemma 3.3. The same argument can be used to prove the rest of part (3).

3.8. The following easy and well known result will be used frequently.

Lemma. Let $R, S$ be rings with a projective right $R$-module $M$ and an $(R, S)$-bimodule $N$ that is projective as a right $S$-module. Then $M \otimes_{R} N$ is a projective right $S$-module. 
3.9. It is now easy to generalise Corollary 3.7 to the modules ${ }_{c+m} \mathrm{P}_{c}$ and ${ }_{c} \mathrm{Q}_{c+m}$ of (1.4.1).

Theorem. Fix $c \in \mathbb{C}$ and an integer $m \geq 1$ such that $c+1, c+2, \ldots, c+m-1$ are all good.

(1) ${ }_{c+m} \mathrm{P}_{c}$ is the unique non-zero $\left(\mathrm{U}_{c+m}, \mathrm{U}_{c}\right)$-bisubmodule of $\mathrm{U}^{\text {reg }}$ that is reflexive as either a right $\mathrm{U}_{c}$-module or a left $\mathrm{U}_{c+m}$-module. Multiplication in $\mathrm{U}^{\mathrm{reg}}$ induces an isomorphism of $\left(\mathrm{U}_{c+m}, \mathrm{U}_{c}\right)$-bimodules,

$$
\left(c+m \mathrm{P}_{c+m-1}\right) \otimes \mathbf{U}_{c+m-1} \cdots \otimes \mathbf{U}_{c+1}\left(c+1 \mathrm{P}_{c}\right) \stackrel{\sim}{\longrightarrow} c+m \mathrm{P}_{c} .
$$

(2) ${ }_{c} \mathrm{Q}_{c+m}$ is the unique nonzero $\left(\mathrm{U}_{c}, \mathrm{U}_{c+m}\right)$-bisubmodule of $\mathrm{U}^{\mathrm{reg}}$ that is reflexive as either a right $\mathrm{U}_{c+m}$-module or a left $\mathrm{U}_{c}$-module. Multiplication gives an isomorphism of $\left(\mathrm{U}_{c}, \mathrm{U}_{c+m}\right)$-bimodules,

$$
\left({ }_{c} \mathrm{Q}_{c+1}\right) \otimes \mathbf{U}_{c+1} \cdots \otimes \mathbf{U}_{c+m-1}\left({ }_{c+m-1} \mathbf{Q}_{c+m}\right) \stackrel{\sim}{\longrightarrow}{ }_{c} \mathbf{Q}_{c+m} .
$$

(3) Either $c$ or $c+m$ is good. In the former case ${ }_{c+m} \mathrm{P}_{c}$ and ${ }_{c} \mathrm{Q}_{c+m}$ are projective $\mathrm{U}_{c}$-modules, while in the latter case they are projective $\mathrm{U}_{c+m}$-modules.

Proof. We only prove assertions for ${ }_{c} \mathrm{Q}_{c+m}$; the proofs for ${ }_{c+m} \mathrm{P}_{c}$ are essentially the same. We will also assume that $c+m$ is good; the proof when $c$ is good is again very similar.

The hypotheses on $c$ now ensure that, by Corollary 3.7, ${ }_{c+i} \mathrm{Q}_{c+i+1}$ is projective as a right $\mathrm{U}_{c+i+1}$-module for all $0 \leq i \leq m-1$. It follows from Lemma 3.8 and a routine induction that (3.9.2) holds and hence that ${ }_{c} \mathrm{Q}_{c+m}$ is both projective and finitely generated as a right $\bigcup_{c+m}$-module. The remaining assertions follow from Theorem 3.5 and Lemma 3.3.

3.10. Example. We end the section by noting that Theorem 3.9 does not extend to all values of $c$. To see this consider the special case when $n=2$ and set $U=U_{\frac{1}{2}}$. Further, let $\bar{U}=U\left(\mathfrak{s l}_{2}\right) /(\Omega)$, where $\Omega$ is the quadratic Casimir element. Using [GS2, Example 6.12] and Remark 2.4, one finds that $U \cong \bar{U} \otimes_{\mathbb{C}} \mathscr{D}\left(\mathbb{A}^{1}\right)$. In this case, $U_{-\frac{1}{2}}$ is a simple ring, necessarily equal to $\operatorname{End}_{U}(Q)$ for $Q={ }_{-\frac{1}{2}} Q_{\frac{1}{2}}$. Write

$$
\operatorname{Tr}(\mathrm{Q})=\left\{\theta(q): \theta \in \operatorname{Hom}_{\mathrm{U}}(Q, \mathrm{U}) \text { and } q \in \mathrm{Q}\right\} \subseteq \mathrm{U}
$$

for the trace ideal of $Q$. Since $U_{-\frac{1}{2}}$ is simple but $U$ is not, the projective $U$-module $\mathrm{Q}$ cannot be a progenerator. Equivalently, $\mathrm{Q}$ is not projective as a left $U_{-\frac{1}{2}}$-module, thereby showing that the final sentence of Theorem 3.9)(3) does not hold for arbitrary $c$.

Write $F$ for the field of fractions of $\mathrm{U}$ and for a $\mathrm{U}$-submodule $M \subset F$, identify $M^{*}=$ $\operatorname{Hom}_{\mathrm{U}}(M, \mathrm{U})$ with $\{\theta \in F: \theta M \subseteq \mathrm{U}\}$. We claim that $V={ }_{-\frac{3}{2}} \mathrm{Q}_{\frac{1}{2}}=\left({ }_{-\frac{3}{2}} \mathrm{Q}_{-\frac{1}{2}}\right) \mathrm{Q}$ is not reflexive on either side. Indeed, suppose that it is reflexive on one side (and hence on both sides by Theorem [3.5). By [St, Theorem B], $\mathrm{U} \cong \mathrm{U}_{-3 / 2}$ has global dimension 2 and so $V$ is also projective on both sides. As $U$ is a maximal order, see [St, Lemma 3.1], $\mathrm{U}_{-3 / 2}=\operatorname{End}_{\mathrm{U}}(V)$ and hence $V^{*}=\operatorname{Hom}_{\mathrm{U}}(V, \mathrm{U})$ is also equal to $\operatorname{Hom}_{\mathrm{U}_{-3 / 2}}\left(V, \mathrm{U}_{-3 / 2}\right)$. 
Thus the Dual Basis Lemma applied to $V$ as a $\mathrm{U}_{-3 / 2^{-m o d u l e}}$ implies that $V^{*} V=\mathrm{U}$. But now $W=V^{*}\left({ }_{-\frac{3}{2}} \mathrm{Q}_{\frac{1}{2}}\right)$ satisfies $W \mathrm{Q}=\mathrm{U}$, contradicting the fact that $\mathrm{Q}$ is not a progenerator. This proves the claim.

\section{Relating hamiltonian REDUCTiOn AND Shift FUnCtors}

4.1. Recall from (2.6) that $\mathfrak{G}=\mathfrak{g} \times V$ with the natural action of $G=G L(V)$ and that, as in (2.6.1), we write

$$
\mathfrak{D}_{c+1}=\frac{\mathscr{D}(\mathfrak{G})}{\mathscr{D}(\mathfrak{G}) \tau\left(I_{c+1}\right)}
$$

thus $\mathfrak{D}_{c+1}^{G} \cong \mathrm{U}_{c}$ by Theorem 2.8. The aim of this section will be to construct a morphism $\Psi_{c}: \mathfrak{D}_{c+1}^{\mathrm{det}^{-1}} \rightarrow{ }_{c-1} \mathrm{Q}_{c}$ and use it to prove Theorem 1.6 in the case $m=1$. The complete proofs of Theorems 1.6 and 1.7, which are given in Section 5, will then follow by applying results from GG]. We begin with the construction of $\Psi_{c}$, for which we need to expand upon the isomorphism in Theorem 2.8 .

4.2. Let vol $\in \wedge^{n} V^{*}$ be a non-zero volume element on $V$ and, following [BFG, Equation 5.3.2], define a map $\mathbf{s}: \mathfrak{G} \rightarrow \mathbb{C}$ by

$$
\mathbf{s}(X, v) \stackrel{\text { def }}{=}\left\langle\text { vol, } v \wedge X v \wedge X^{2} v \wedge \cdots \wedge X^{n-1} v\right\rangle .
$$

Clearly $\mathbf{s} \neq 0$ and, as $(g \cdot \mathbf{s})(X, v)=\mathbf{s}\left(g^{-1} \cdot(X, v)\right)=\mathbf{s}\left(g^{-1} X g, g^{-1} v\right)$, it is $\operatorname{a~det}^{-1}$ semi-invariant under the $G L(V)$-action 11 Note also that $\mathbf{s}_{\mid \mathfrak{h}^{\text {reg }}}=\delta$.

We now want to use the radial component map, described as follows. Take the Zariski open dense subset

$$
\mathfrak{G}^{\text {cyc }} \stackrel{\text { def }}{=}\{(X, v): v \text { is a cyclic vector for the operator } X: V \rightarrow V\} \subset \mathfrak{G}
$$

and, for $c \in \mathbb{C}$, let

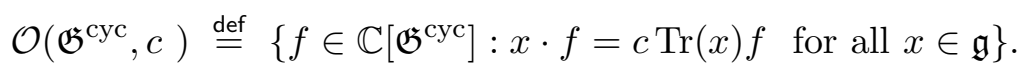

By [BFG, Corollary 5.3.4] restriction of functions induces an isomorphism $\mathcal{O}\left(\mathfrak{G}^{\text {cyc }}, c\right) \cong$ $\mathbf{s}^{-c} \mathbb{C}[\mathfrak{h} / W]$. Moreover, if $D \in \mathscr{D}\left(\mathfrak{G}^{c y c}\right)^{G}$ then, by restriction, $D$ induces a differential operator from $\mathcal{O}\left(\mathfrak{G}^{\text {cyc }}, c\right)$ to itself. This defines the twisted radial components map

$$
\mathfrak{R}_{c}: \mathscr{D}\left(\mathfrak{G}^{\mathrm{cyc}}\right)^{G} \longrightarrow \mathscr{D}(\mathfrak{h} / W), \quad D \longmapsto \mathfrak{R}_{c}(D)=\mathbf{s}^{c} \circ\left(\left.D\right|_{\mathcal{O}\left(\mathfrak{G}^{\mathrm{cyc}}, c\right)}\right) \circ \mathbf{s}^{-c} .
$$

As in [GG, Section 6.5] and in the notation of (2.6.1), this map is an algebra homomorphism that induces the isomorphism

$$
\overline{\mathfrak{R}}_{c}: \mathfrak{D}_{c}^{G}=\left(\mathscr{D}(\mathfrak{G}) / \mathscr{D}(\mathfrak{G}) \tau\left(I_{c}\right)\right)^{G} \stackrel{\sim}{\rightarrow} \mathrm{U}_{c-1}
$$

from Theorem 2.8. The details are given in the appendix to this paper. In particular, $\mathfrak{R}_{c}$ restricts to give a surjection $\mathscr{D}(\mathfrak{G})^{G} \rightarrow \mathrm{U}_{c-1}$.

\footnotetext{
${ }^{1}$ As we mentioned in Section 2.1. $[\mathrm{BFG}$ ] and $\mathrm{GG}$ ] use the opposite convention for group actions and so in those papers s transforms by the determinant; see, for example, the sentence after GG, (6.18)].
} 
As is observed after [GG, (6.18)], $\mathfrak{G}^{\text {cyc }}=\mathfrak{G} \backslash \mathbf{s}^{-1}(0)$ and so, if $D \in \mathscr{D}(\mathfrak{G})^{\operatorname{det}^{-1}}$, then $D \mathbf{s}^{-1}$ is a well-defined $G$-invariant differential operator on $\mathbb{C}\left[\mathfrak{G}^{\text {cyc }}\right]$. Hence we obtain a map

$$
\widehat{\Psi}_{c}: \mathscr{D}(\mathfrak{G})^{\operatorname{det}^{-1}} \longrightarrow e \mathscr{D}(\mathfrak{h} / W) e \subset \mathrm{U}^{\mathrm{reg}}=e \mathscr{D}\left(\mathfrak{h}^{\mathrm{reg}}\right)^{W} e, \quad D \longmapsto e \mathfrak{R}\left(D \mathbf{s}^{-1}\right) e
$$

As in the proof of Lemma 2.2, we have $\left[\tau(x), \mathbf{s}^{-1}\right]=\operatorname{Tr}(x) \mathbf{s}^{-1}$ for any $x \in \mathfrak{g}$ and so

$$
(\tau(x)-(c+1) \operatorname{Tr}(x)) \mathbf{s}^{-1}=\mathbf{s}^{-1}(\tau(x)-c \cdot \operatorname{Tr}(x)) \in \mathscr{D}\left(\mathfrak{G}^{\mathrm{cyc}}\right) \tau\left(I_{c}\right) .
$$

Therefore the map $\widehat{\Psi}_{c}$ factors through $\mathfrak{D}_{c+1}^{\text {det }^{-1}}$ to give the desired map

$$
\Psi_{c}: \mathfrak{D}_{c+1}^{\mathrm{det}^{-1}} \rightarrow \mathrm{U}^{\mathrm{reg}} .
$$

Lemma. Under multiplication in $\mathrm{U}^{\mathrm{reg}}$, the image $\operatorname{Im}\left(\Psi_{c}\right)$ is a non-zero $\left(U_{c-1}, U_{c}\right)$ bimodule. Moreover, $\Psi_{c}$ is a $\left(\mathrm{U}_{c-1}, \mathrm{U}_{c}\right)$-bimodule homomorphism in the sense that

$$
\Psi_{c}\left(\alpha_{c} \beta \alpha_{c+1}\right)=\bar{\Re}_{c}\left(\alpha_{c}\right) \Psi_{c}(\beta) \overline{\mathfrak{R}}_{c+1}\left(\alpha_{c+1}\right)
$$

for $\alpha_{c} \in \mathfrak{D}_{c}^{G}, \alpha_{c+1} \in \mathfrak{D}_{c+1}^{G}$ and $\beta \in \mathfrak{D}_{c+1}^{\operatorname{det}^{-1}}$.

Proof. Note that $\mathbf{s} \in \mathscr{D}(\mathfrak{G})^{\operatorname{det}^{-1}}$ and that $\Psi_{c}([\mathbf{s}])=e \mathfrak{R}\left(\mathbf{s}^{-1} \mathbf{s}\right) e=e$. Hence $\Psi_{c} \neq 0$. Combined with (4.2.3), it now suffices to prove (4.2.5).

By Lemma 2.2, $\alpha_{c} \beta \alpha_{c+1} \in \mathfrak{D}_{c+1}^{\mathrm{det}^{-1}}$ and so the left hand side of (4.2.5) is well defined. So (4.2.5) therefore follows from the computation

$$
\begin{aligned}
\overline{\mathfrak{R}}_{c}\left(\alpha_{c}\right) \Psi_{c}(\beta) \overline{\mathfrak{R}}_{c+1}\left(\alpha_{c+1}\right) & =\mathbf{s}^{c} \alpha_{c} \mathbf{s}^{-c} \cdot \mathbf{s}^{c}\left(\beta \mathbf{s}^{-1}\right) \mathbf{s}^{-c} \cdot \mathbf{s}^{c+1} \alpha_{c+1} \mathbf{s}^{-(c+1)} \\
& =\mathbf{s}^{c}\left(\alpha_{c} \beta \alpha_{c+1} \mathbf{s}^{-1}\right) \mathbf{s}^{-c}=\Psi_{c}\left(\alpha_{c} \beta \alpha_{c+1}\right) .
\end{aligned}
$$

4.3. Let $\mu_{\mathfrak{G}}: T^{*} \mathfrak{G} \rightarrow \mathfrak{g}^{*} \cong \mathfrak{g}$ be the moment map as defined in [GG, (2.4)] and, as in GG, (1.1)], set

$$
\mathcal{M} \stackrel{\text { def }}{=} \mu_{\mathfrak{G}}^{-1}(0)=\left\{(X, Y, v, w) \in \mathfrak{g} \times \mathfrak{g} \times V \times V^{*} \mid[X, Y]+v w=0\right\} .
$$

Recall that $A^{1}=\mathbb{C}\left[\mathfrak{h} \times \mathfrak{h}^{*}\right]^{\text {sign }}$. The powers $A^{r}$ are obtained by multiplication inside $\mathbb{C}\left[\mathfrak{h} \times \mathfrak{h}^{*}\right]$ : they will be regarded as modules over $A^{0}=\mathbb{C}\left[\mathfrak{h} \times \mathfrak{h}^{*}\right]^{W}$ under the natural induced structure. Then, once one recalls our conventions about group actions from 2.1, it follows from [GG, Proposition A2] that

$$
A^{r} \cong \mathbb{C}[\mathcal{M}]^{\operatorname{det}^{-r}}, \quad r=0,1,2, \ldots
$$

This result is stated in [GG] as an isomorphism of vector spaces, but the proof shows that it is in fact an isomorphism of graded $A^{0}$-modules. 
4.4. As before, for any subfactor $T$ of $\mathscr{D}(\mathfrak{G})$ with the induced differential operator filtration, we write $\operatorname{gr} T=\operatorname{gr}_{\Gamma} T$ for the associated graded object. We will consider $\mathbb{C}\left[\mathfrak{h} \times \mathfrak{h}^{*}\right]$ and its submodules as graded in the second term; equivalently they are given the gradation induced from the identity $\mathbb{C}\left[\mathfrak{h} \times \mathfrak{h}^{*}\right]=\operatorname{gr} \mathscr{D}(\mathfrak{h})$. Recall that, by Theorem 2.8 and (4.2.3), the map $\mathfrak{R}_{c}$ induces a graded isomorphism gr $\overline{\mathfrak{R}}: \operatorname{gr} \mathfrak{D}_{c}^{G} \stackrel{\sim}{\longrightarrow} A^{0}=\mathbb{C}\left[\mathfrak{h} \times \mathfrak{h}^{*}\right]^{W}$.

Lemma. (1) For all $c \in \mathbb{C}$, and $r \in \mathbb{N}$, we have gr $\mathfrak{D}_{c+1}^{\mathrm{det}^{-r}} \cong A^{r}$ as graded $A^{0}$-modules, where the $A^{0}$-module structure of gr $\mathfrak{D}_{c+1}^{\mathrm{det}^{-r}}$ is defined via gr $\bar{\Re}$.

(2) For all $c \in \mathbb{C}, \mathfrak{D}_{c+1}^{\mathrm{det}^{-1}}$ is reflexive as both a right $\mathrm{U}_{c}$-module and a left $\mathrm{U}_{c-1}$-module.

Proof. (1) We note for future reference that the action of $G$ preserves the differential operator filtration and so, for any $r \in \mathbb{Z}$, we have $\operatorname{gr}\left(\mathscr{D}(\mathfrak{G})^{\operatorname{det}^{r}}\right)=(\operatorname{gr} \mathscr{D}(\mathfrak{G}))^{\operatorname{det}^{r}}$. In particular we can identify

$$
\left(\operatorname{gr} \mathfrak{D}_{c+1}\right)^{\operatorname{det}^{-r}}=\frac{\operatorname{gr}\left(\mathscr{D}(\mathfrak{G})^{\operatorname{det}^{-r}}\right)}{\operatorname{gr}\left(\left[\mathscr{D}(\mathfrak{G}) \tau\left(I_{c+1}\right)\right]^{\operatorname{det}^{-r}}\right)}
$$

without causing ambiguity.

We return to the proof and write $\mu=\mu_{\mathfrak{G}}$. By construction there is a sequence of graded $\mathbb{C}\left[\mathfrak{h} \times \mathfrak{h}^{*}\right]^{W}$-module maps:

$$
\begin{aligned}
\chi: A^{r} \cong \mathbb{C}[\mathcal{M}]^{\operatorname{det}^{-r}}= & {\left[\frac{\mathbb{C}\left[T^{*} \mathfrak{G}\right]}{\mathbb{C}\left[T^{*} \mathfrak{G}\right] \cdot \mu^{*}(\mathfrak{g})}\right]^{\operatorname{det}^{-r}}=\left[\frac{\operatorname{gr} \mathscr{D}(\mathfrak{G})}{\operatorname{gr} \mathscr{D}(\mathfrak{G}) \cdot \operatorname{gr} \tau\left(I_{c+1}\right)}\right]^{\operatorname{det}^{-r}} } \\
& \stackrel{\alpha}{\longrightarrow}\left[\frac{\operatorname{gr} \mathscr{D}(\mathfrak{G})}{\operatorname{gr}\left(\mathscr{D}(\mathfrak{G}) \tau\left(I_{c+1}\right)\right)}\right]^{\operatorname{det}^{-r}}
\end{aligned}
$$

By [GG, Corollary 2.6] $\mu$ is flat and so, by [Ho, Proposition 2.4], the final surjection $\alpha$ in (4.4.1) is an isomorphism. Hence $\chi$ is an isomorphism.

(2) The fact that $\mathfrak{D}_{c+1}^{\mathrm{det}^{-1}}$ is a $\left(\mathrm{U}_{c-1}, \mathrm{U}_{c}\right)$-bimodule is part of Lemma 4.2. By part (1), $\operatorname{gr} \mathfrak{D}_{c+1}^{\text {det }^{-1}} \cong A^{1}$ as both a right module over gr $\bigcup_{c} \cong \mathbb{C}\left[\mathfrak{h} \times \mathfrak{h}^{*}\right]^{W}$ and as a left module over gr $\bigcup_{c-1} \cong \mathbb{C}\left[\mathfrak{h} \times \mathfrak{h}^{*}\right]^{W}$. The proof of Corollary $3.7(2)$ can now be used unchanged to give the desired result.

4.5. We are now able to prove Theorem 1.6 in the case $m=1$ and we are able to do so without restriction on $c$. Thus we prove:

Proposition. For any $c \in \mathbb{C}$ the homomorphism $\Psi_{c}$ from (4.2.4) induces an isomorphism $\mathfrak{D}_{c+1}^{\text {det }^{-1}} \cong{ }_{c-1} \mathrm{Q}_{c}$ of $\left(\mathrm{U}_{c-1}, \mathrm{U}_{c}\right)$-bimodules.

Proof. A key observation here is that $\mathrm{U}_{c}$ is a noetherian domain with quotient division ring $F$ containing $\mathrm{U}^{\mathrm{reg}}$. Hence any non-zero finitely generated $\mathrm{U}_{c^{-}}$-submodule $M$ of $F$ must be a torsion-free module of Goldie rank one; equivalently, every proper factor of $M$ is a torsion module. 
We first claim that $\Psi_{c}$ is injective. To see this note that, by Lemma 4.4 (1), gr $\mathfrak{D}_{c+1}^{\text {det }^{-1}}$ is a torsion-free, rank one module over the domain $\operatorname{gr}\left(\mathrm{U}_{c}\right) \cong \mathbb{C}\left[\mathfrak{h} \times \mathfrak{h}^{*}\right]^{W}$. Consequently, $\mathfrak{D}_{c+1}^{\text {det }^{-1}}$ is a torsion-free right $U_{c}$-module of Goldie rank one and so any proper factor of this module would be torsion (or zero). But, by Lemma 4.2, $\operatorname{Im}\left(\Psi_{c}\right) \subset \mathrm{U}^{\text {reg }}$ is a non-zero torsion-free $\mathrm{U}_{c}$-module. Hence $\Psi_{c}$ is indeed injective.

Therefore, by Lemma 4.4(2), respectively Corollary 3.7(2), both $\operatorname{Im}\left(\Psi_{c}\right)$ and ${ }_{c-1} \mathrm{Q}_{c}$ are reflexive $\left(\mathrm{U}_{c-1}, \mathrm{U}_{c}\right)$-bimodules of $\mathrm{U}^{\mathrm{reg}}$, and we emphasise that in both cases the bimodule structure is that induced from the bimodule structure of $U^{\text {reg. By }}$ Theorem 3.5 and Lemma 3.3 they are therefore equal.

4.6. We end the section by strengthening the isomorphism from Proposition 4.5 to an isomorphism of filtered spaces. The difficulty here is that, although $\mathfrak{D}_{c+1}^{\mathrm{det}^{-1}}$ and ${ }_{c-1} \mathrm{Q}_{c}$ are given the differential operator filtrations $\Gamma$, these are induced from two different rings of differential operators $\mathscr{D}(\mathfrak{G})$, respectively $\mathscr{D}\left(\mathfrak{h}^{\text {reg }}\right)$. In the abstract it is not clear that the two resulting filtrations on ${ }_{c-1} Q_{c}$ are closely related (see [Le, Problème 3.5], for example). Fortunately, this problem is easily resolved in the present setting.

Corollary. Keep the notation of Proposition 4.5. Then the isomorphism $\Psi_{c}$ is a filtered isomorphism in the sense that $\Psi_{c}\left(\Gamma_{r} \mathfrak{D}_{c+1}^{\operatorname{det}^{-1}}\right)=\Gamma_{r}\left({ }_{c-1} \mathrm{Q}_{c}\right)$ for all $r$.

Proof. Set $D=\mathfrak{D}_{c+1}^{\operatorname{det}^{-1}}$ and $Q={ }_{c-1} \mathrm{Q}_{c}$. The analogous isomorphism of filtered algebras $\mathfrak{D}_{c+1}^{G} \cong \mathrm{U}_{c}$ is given by Theorem 2.8. Since the radial component map $\mathfrak{R}$ at worst decreases the degree of an operator, the map $\Psi_{c}$ is at least a map of filtered modules in the sense that $\Psi_{c}\left(\Gamma_{m} D\right) \subseteq \Gamma_{m} Q$ for all $m \geq 0$. Suppose that $\Psi_{c}$ is not an isomorphism of filtered objects. Since $\Psi_{c}$ is an isomorphism of unfiltered modules, there must then exist some $d \in D$ for which the order of $\Psi_{c}(d)$ is less than the order of $d$. Hence $\operatorname{gr}\left(\Psi_{c}\right)$ : $\operatorname{gr}_{\Gamma} D \rightarrow \operatorname{gr}_{\Gamma} Q$ will have a nonzero kernel. But we know from Lemma 4.4 (1) that $\operatorname{gr}_{\Gamma} D$ is a rank one torsion-free $\mathbb{C}\left[\mathfrak{h} \times \mathfrak{h}^{*}\right]^{W}$-module, and so its image must therefore be torsion. Since $\operatorname{gr}_{\Gamma} Q$ is a submodule of the domain $\operatorname{gr}_{\Gamma} \mathrm{U}^{\text {reg }}=\mathbb{C}\left[\mathfrak{h}^{\text {reg }} \times \mathfrak{h}^{*}\right]^{W}$ this forces $\operatorname{gr}(\Psi)=0$. This contradicts the fact that, for example, $0 \neq e=\Psi_{c}(\mathbf{s}) \in \Psi\left(\Lambda_{0} D\right) \subseteq \Gamma_{0} Q$.

\section{Proof of Theorems 1.6 and 1.7}

5.1. We continue to write $\mathfrak{D}_{c}=\mathscr{D}(\mathfrak{G}) / \mathscr{D}(\mathfrak{G}) \tau\left(I_{c}\right)$ in the notation of (2.6.1), and recall from Theorem 2.8 that $\mathfrak{D}_{c}^{G} \cong \mathrm{U}_{c-1}$. For any $p, q \in \mathbb{Z}_{\geq 0}$, multiplication on $\mathscr{D}(\mathfrak{G})$ induces a map

$$
\mathscr{D}(\mathfrak{G})^{\operatorname{det}^{-p}} \otimes_{\mathscr{D}(\mathfrak{G})^{G}} \mathscr{D}(\mathfrak{G})^{\operatorname{det}^{-q}} \rightarrow \mathscr{D}(\mathfrak{G})^{\operatorname{det}^{-(p+q)}}
$$

It follows from Lemma 2.2 that, for any $a \in \mathbb{C}$, this factors to give a multiplication map of $\left(\mathfrak{D}_{a-q-p}^{G}, \mathfrak{D}_{a}^{G}\right)$-bimodules:

$$
\mu_{p, q}:\left(\mathfrak{D}_{a-q}\right)^{\operatorname{det}^{-p}} \otimes_{\mathfrak{D}_{a-q}^{G}}\left(\mathfrak{D}_{a}\right)^{\operatorname{det}^{-q}} \longrightarrow\left(\mathfrak{D}_{a}\right)^{\operatorname{det}^{-(p+q)}} .
$$


The aim of this section is to thoroughly understand this: for the appropriate values of $c$, we will show that $\mathfrak{D}_{c-q}^{\text {det }^{-p}} \cong{ }_{c-p-q-1} \mathrm{Q}_{c-q-1}$ as a $\left(\mathrm{U}_{c-p-q}, \mathrm{U}_{c-q-1}\right)$-bimodule and that

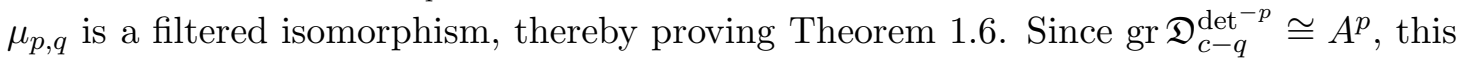
will also prove Theorem 1.7(1), from which the rest of that theorem follows easily. As might be expected, the proof is by induction on $p, q$ with the results of the last section providing the starting point.

5.2. Most modules considered in this paper are subfactors of rings of differential operators and, unless we say otherwise, they will then be given the differential operator filtration induced from that ambient space. One exception is with tensor products. We recall that if $R=\bigcup R_{i}$ and $S=\bigcup S_{j}$ are filtered modules over some ring $U$ then the tensor product filtration $\Lambda$ on $R \otimes_{U} S$ is given by $\Lambda_{n}\left(R \otimes_{U} S\right)=\sum_{i+j=n} R_{i} \otimes S_{j}$.

Lemma. Let $p, q \in \mathbb{N}$, set $r=p+q$, and pick $a \in \mathbb{C}$ such that $a-2, a-3, \ldots, a-r$ are all good (this is automatic if $r=0$ or 1 ).

(1) If $r>0$ then either $a-1$ or $a-r-1$ is good. In the former case $\mathfrak{D}_{a}^{\operatorname{det}^{-r}}$ is a projective right $\mathfrak{D}_{a}^{G}$-module while in the latter it is a projective left $\mathfrak{D}_{a-r}^{G}$-module.

(2) With the tensor product filtration on the left hand side of (5.1.1), the map $\mu_{p, q}$ is a filtered $\left(\mathfrak{D}_{a-p-q}^{G}, \mathfrak{D}_{a}^{G}\right)$-bimodule isomorphism.

Proof. We prove the two parts of the lemma by simultaneous induction on $r$. Note that (1) is vacuous if $r=0$ and that (2) is automatic if either $p=0$ or $q=0$. Suppose that $r=p+q=1$. Then Proposition 4.5 implies that $\mathfrak{D}_{a}^{\mathrm{det}^{-1}} \cong{ }_{a-2} \mathrm{Q}_{a-1}$ as $\left(\mathrm{U}_{a-2}, \mathrm{U}_{a}\right)$ bimodules and so (1) is given by Theorem 3.9(3).

We may now assume that $0<p, q<r$. This ensures that $a-q-1$ is good and so the induction hypothesis implies that both $\mathfrak{D}_{a-q}^{\operatorname{det}^{-p}}$ and $\mathfrak{D}_{a}^{\text {det }^{-q}}$ are projective modules over $\mathfrak{D}_{a-q}^{G}$. Moreover, as one of $a-r-1$ and $a-1$ is good, one of these modules is also a projective module on the other side. Thus, once the proof of part (2) is complete, part (1) will follow from Lemma 3.8 .

It remains to prove part (2). We first claim that $\mathfrak{D}_{a-q}^{\operatorname{det}^{-p}} \otimes_{\mathfrak{D}_{a-q}^{G}} \mathfrak{D}_{a}^{\operatorname{det}^{-q}}$ is a rank one, torsion-free right $\mathfrak{D}_{a}^{G}$-module. To see this, first take the differential operator filtration

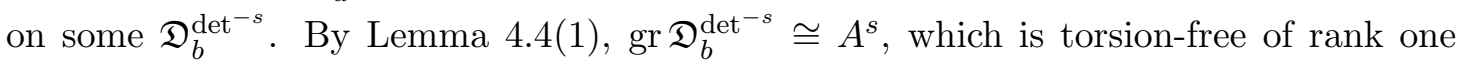
as a module over $A^{0}=\mathbb{C}\left[\mathfrak{h} \times \mathfrak{h}^{*}\right]^{W}=\operatorname{gr} \mathfrak{D}_{b}^{G}$. It follows that $\mathfrak{D}_{b}^{\operatorname{det}^{-s}}$ is a rank one torsion-free right $\mathfrak{D}_{b}^{G}$-module; in particular $\mathfrak{D}_{a-q}^{\operatorname{det}^{-p}}$ and $\mathfrak{D}_{a}^{\operatorname{det}^{-q}}$ are rank one torsionfree modules on the right. By the previous paragraph they are also both projective modules over $\mathfrak{D}_{a-q}^{G}$. Hence, as a right $\mathfrak{D}_{a}^{G}$-module, $\mathfrak{D}_{a-q}^{\mathrm{det}^{-p}} \otimes_{\mathfrak{D}_{a-q}^{G}} \mathfrak{D}_{a}^{\mathrm{det}^{-q}}$ embeds into $\mathfrak{D}_{a-q}^{G} \otimes_{\mathfrak{D}_{a-q}^{G}} \mathfrak{D}_{a}^{\text {det }^{-q}}=\mathfrak{D}_{a}^{\text {det }^{-q}}$, which is a rank one torsion-free right $\mathfrak{D}_{a}^{G}$-module. This proves the claim.

We return to the proof of part (2). It is clear from Lemma 2.2 and Theorem 2.8 that $\mu_{p, q}$ is a $\left(\mathfrak{D}_{a-p-q}^{G}, \mathfrak{D}_{a}^{G}\right)$-bimodule homomorphism and so we just need to prove that it is a filtered vector space isomorphism. We first show that $\mu_{p, q}$ injective. To see this 
note that, as above, $\mathfrak{D}_{a}^{\text {det }^{-r}}$ is a torsion-free right $\mathfrak{D}_{a}^{G}$-module. Also, $\operatorname{Im}\left(\mu_{p, q}\right) \neq 0$ since $\mu_{p, q}\left(\mathbf{s}^{p} \otimes \mathbf{s}^{q}\right)=\mathbf{s}^{p+q} \neq 0$. Hence $\operatorname{Im}\left(\mu_{p, q}\right)$ is a non-zero torsion-free right $\mathfrak{D}_{a}^{G}$-module. Since $\mathfrak{D}_{a-q}^{\operatorname{det}^{-p}} \otimes_{\mathfrak{D}_{a-q}^{G}} \mathfrak{D}_{a}^{\text {det }^{-q}}$ is a rank one torsion-free right $\mathfrak{D}_{a}^{G}$-module, this forces $\mu_{p, q}$ to be injective.

We next prove that $\mu_{p, q}$ is surjective. We can now identify $\mathfrak{D}_{a-q}^{\operatorname{det}^{-p}} \otimes_{\mathfrak{D}_{a-q}^{G}} \mathfrak{D}_{a}^{\operatorname{det}^{-q}}$ with its image $\mathfrak{D}_{a-q}^{\operatorname{det}^{-p}} \cdot \mathfrak{D}_{a}^{\operatorname{det}^{-q}}$ under $\mu_{p, q}$. This is filtered by the image of the tensor product filtration and so [GS1, Lemma 6.7(1)] implies that

$$
\operatorname{gr}\left(\mathfrak{D}_{a-q}^{\operatorname{det}^{-p}}\right) \cdot \operatorname{gr}\left(\mathfrak{D}_{a}^{\operatorname{det}^{-q}}\right) \subseteq \operatorname{gr}\left(\mathfrak{D}_{a}^{\operatorname{det}^{-r}}\right) .
$$

By Lemma 4.4(1) we can regard this multiplication as taking place inside $\mathbb{C}\left[\mathfrak{h} \times \mathfrak{h}^{*}\right]$ in which case two further applications of that lemma give

$$
\left(\operatorname{gr} \mathfrak{D}_{a-q}^{\operatorname{det}^{-p}}\right) \cdot\left(\operatorname{gr} \mathfrak{D}_{a}^{\operatorname{det}^{-q}}\right)=A^{p} \cdot A^{q}=A^{p+q}=\operatorname{gr} \mathfrak{D}_{a}^{\operatorname{det}^{-r}} .
$$

Thus $\mu_{p, q}$ is graded surjective and hence surjective. Since it is immediate that $\mu_{p, q}$ is a filtered homomorphism, this also proves that it is a filtered isomorphism. This completes the proof of part (2) and hence of the lemma.

5.3. We can now transfer results from the gr $\mathfrak{D}_{c}^{\operatorname{det}^{-p}}$ to the ${ }_{c-p-1} \mathrm{Q}_{c-1}$ and complete the proofs of Theorems 1.6 and 1.7 for the modules ${ }_{a} \mathrm{Q}_{b}$.

Theorem. Fix an integer $m \geq 0$ and $c \in \mathbb{C}$ such that the numbers $c-1, c-2, \ldots, c-m+1$ are good (this is automatic if $m=0,1$ ).

(1) Under the differential operator filtration on both sides there is a filtered isomorphism $\Theta_{c, m}: \mathfrak{D}_{c+1}^{\operatorname{det}^{-m}} \stackrel{\sim}{\longrightarrow}{ }_{c-m} \mathbf{Q}_{c}$ of $\left(\mathbf{U}_{c-m}, \mathbf{U}_{c}\right)$-bimodules.

(2) Under the differential operator filtration $\Gamma$ we have $\operatorname{gr}_{\Gamma}{ }_{c-m} Q_{c}=\delta^{-m} A^{m} e$.

Proof. When $m=0,1$, the result follows from Theorem 2.8, respectively Corollary 4.6 and Lemma 4.4. So we can assume that $m \geq 2$.

Consider the following chain of maps:

$$
\begin{aligned}
\Theta: \mathfrak{D}_{c+1}^{\mathrm{det}^{-m}} & \stackrel{\alpha}{\longrightarrow} \mathfrak{D}_{c-m+2}^{\mathrm{det}^{-1}} \otimes_{\mathfrak{D}_{c-m+2}^{G}} \cdots \otimes_{\mathfrak{D}_{c}^{G}} \mathfrak{D}_{c+1}^{\mathrm{det}^{-1}} \\
& \stackrel{\beta}{\longrightarrow}\left({ }_{c-m} \mathrm{Q}_{c-m+1}\right) \otimes_{\mathbf{U}_{c-m+1}} \cdots \otimes_{\mathbf{U}_{c-1}}\left({ }_{c-1} \mathbf{Q}_{c}\right) \stackrel{\gamma}{\longrightarrow}{ }_{c-m} \mathbf{Q}_{c} .
\end{aligned}
$$

Here $\alpha$ is the isomorphism given by iterating the $\mu_{p q}^{-1}$ for appropriate $p, q$ and applying Lemma $5.2(2)$. Similarly, $\beta$ is the map $\Psi_{c-m+1} \otimes \cdots \otimes \Psi_{c}$, which is an isomorphism by Proposition 4.5 and induction. Finally, by (3.9.2) $\gamma$ is an isomorphism induced by multiplication in $\mathbf{U}^{\text {reg }}$. By those same references, each of these maps is a $\left(\mathbf{U}_{c-m}, \mathbf{U}_{c}\right)$ bimodule map and hence $\Theta$ is a $\left(\mathrm{U}_{c-m}, \mathrm{U}_{c}\right)$-bimodule isomorphism.

We claim that $\Theta$ is a filtered isomorphism, where the domain and codomain are given the filtrations $\Gamma$ induced by the differential operator filtration in $\mathscr{D}(\mathfrak{G})$ and $\mathscr{D}\left(\mathfrak{h}^{\text {reg }}\right) * W$ respectively. On the two middle terms we will take the tensor product filtration induced from the differential operator filtration on the individual tensor-summands. 
In order to prove this claim, we study the individual maps. By Lemma 5.2(2) each map $\mu_{p, q}$ is a filtered isomorphism and hence so is $\mu_{p, q}^{-1}$. By induction this implies that $\alpha$ is also a filtered isomorphism. If we take the tensor product filtration on both

$$
\mathfrak{D}_{c-m+2}^{\text {det }^{-1}} \otimes \cdots \otimes \mathfrak{D}_{c+1}^{\text {det }^{-1}} \quad \text { and } \quad{ }_{c-m} \mathbf{Q}_{c-m+1} \otimes \cdots \otimes{ }_{c-1} \mathbf{Q}_{c},
$$

then Corollary 4.6 implies that $\beta$ is then a filtered isomorphism. Next, it is clear that $\gamma$ is a map of filtered modules since

$$
\begin{aligned}
& \gamma\left(\Gamma_{r_{1}}\left(c-m \mathrm{Q}_{c-m+1}\right) \otimes \cdots \otimes \Gamma_{r_{m}}\left({ }_{c-1} \mathrm{Q}_{c}\right)\right) \\
& \quad=\Gamma_{r_{1}}\left({ }_{c-m} \mathrm{Q}_{c-m+1}\right) \cdots \Gamma_{r_{m}}\left({ }_{c-1} \mathrm{Q}_{c}\right) \subseteq \Gamma_{r_{1}+\cdots+r_{m}}\left({ }_{c-m} \mathrm{Q}_{c}\right),
\end{aligned}
$$

for all $r_{i} \geq 0$. However, we do not yet know that $\gamma$ is a filtered isomorphism.

Putting these facts together implies that $\Theta$ is a filtered homomorphism. We now follow the argument of Corollary 4.6 to deduce that $\Theta$ is a filtered isomorphism. Suppose this is not true. Then, as $\Theta$ is an isomorphism of unfiltered objects, there must then exist some $d \in D=\mathfrak{D}_{c}^{\text {det }^{-m}}$ for which the order of $\Theta(d)$ is strictly less than the order of $d$. Hence $\operatorname{gr}(\Theta): \operatorname{gr}_{\Gamma} D \rightarrow \operatorname{gr}_{\Gamma}\left({ }_{c-m} \mathrm{Q}_{c}\right)$ will have a nonzero kernel. But we know from Lemma 4.4(1) that $\operatorname{gr}_{\Gamma} D$ is a rank one torsion-free $\mathbb{C}\left[\mathfrak{h} \times \mathfrak{h}^{*}\right]^{W}$-module, and so its image must therefore be torsion. Since $\operatorname{gr}_{\Gamma}\left({ }_{c-m} \mathrm{Q}_{c}\right)$ is a submodule of the torsion-free $A^{0}$ module $\operatorname{gr}_{\Gamma} \mathrm{U}^{\text {reg }}=\mathbb{C}\left[\mathfrak{h}^{\text {reg }} \times \mathfrak{h}^{*}\right]^{W} e$ this forces $\operatorname{gr}(\Theta)=0$. This contradicts the fact that, for example, $0 \neq e=\Theta\left(\mathbf{s}^{m}\right) \in \Theta\left(\Gamma_{0} D\right) \subseteq \Gamma_{0}\left({ }_{c-m} \mathbf{Q}_{c}\right)$, where $\mathbf{s}$ is the function defined in (4.2.1). This proves part (1) of the theorem.

It now follows from Lemma 4.4 that $\operatorname{gr}_{\Gamma}\left({ }_{c-m} \mathrm{Q}_{c}\right) \cong \operatorname{gr}_{\Gamma} \mathfrak{D}_{c+1}^{\text {det }^{-m}} \cong A^{m} \cong \delta^{-m} A^{m} e$ as graded $A^{0}$-modules, thereby proving that part (2) holds up to isomorphism. However, the theorem asserts that there is an equality of graded modules $\operatorname{gr}_{\Gamma}\left({ }_{c-m} \mathbf{Q}_{c}\right)=e \delta^{-m} A^{m}$ as subspaces of $\operatorname{gr}_{\Gamma} \mathrm{U}^{\text {reg }}=e \mathbb{C}\left[\mathfrak{h}^{\text {reg }} \times \mathfrak{h}^{*}\right]^{W}$. This requires a little more work.

We prove this equality by induction. As in the proof of [GS1, Lemma 6.9(2)], and for any $a \in \mathbb{C}$,

$$
\operatorname{gr}_{\Gamma}\left({ }_{a-1} \mathbf{Q}_{a}\right)=\operatorname{gr}_{\Gamma}\left(e \delta^{-1} H_{a} e\right)=e \delta^{-1} \mathbb{C}\left[\mathfrak{h} \times \mathfrak{h}^{*}\right] * W e=e \delta^{-1} \mathbb{C}\left[\mathfrak{h} \times \mathfrak{h}^{*}\right]^{\text {sign }} e=e \delta^{-1} A^{1},
$$

and so the result holds for $m=1$. Since we have proved that both $\alpha$ and $\beta$ are filtered isomorphisms, the fact that $\Theta$ is a filtered isomorphism also implies that $\gamma$ is a filtered isomorphism. This can be tautologically reformulated as the statement:

the differential operator and tensor product filtrations are equal on

$$
{ }_{c-m} \mathrm{Q}_{c}=\left({ }_{c-m} \mathrm{Q}_{c-m+1}\right) \cdot\left({ }_{c-m+1} \mathrm{Q}_{c-m+2}\right) \cdots\left({ }_{c-1} \mathrm{Q}_{c}\right) .
$$

By [GS1, Lemma 6.7(2)] and induction, the multiplication map therefore induces a surjection

$$
\chi: e \delta^{-m} A^{m}=\operatorname{gr}_{\Gamma}\left(c-m \mathrm{Q}_{c-m+1}\right) \cdots \operatorname{gr}_{\Gamma}\left({ }_{c-1} \mathrm{Q}_{c}\right) \longrightarrow \operatorname{gr}_{\Gamma}\left({ }_{c-m} \mathrm{Q}_{c}\right) .
$$

As both sides of this equation are non-zero rank one torsion-free $A^{0}$-modules, $\chi$ must be an equality. 
5.4. We can use the theorem to improve the results from Lemma 5.2 .

Corollary. Fix an integer $m>0$ and $c \in \mathbb{C}$ such that $c-1, c-2, \ldots, c-m+1$ are all good. Then, $\mathfrak{D}_{c+1}^{\mathrm{det}^{-m}}$ is reflexive as both a left $\mathfrak{D}_{c-m+1}^{G}$-module and a right $\mathfrak{D}_{c+1}^{G}$-module and is the unique such reflexive bisubmodule of $\mathrm{U}^{\mathrm{reg}}$.

Proof. Combine Theorems 5.3 and $3.9(2)$.

5.5. Remark. Theorem 5.3 completes the proof of Theorem 1.6 and Theorem 1.7(1).

In order to complete the proof of Theorem 1.7 we need to understand the associated graded modules of the ${ }_{c+m} \mathrm{P}_{c}$. We expect, but have not pursued, an isomorphism analogous to Theorem $\left[5.3(1)\right.$ between ${ }_{c+m} \mathrm{P}_{c}$ and $\left[\mathscr{D}(\mathfrak{G}) / I_{c+m+1} \mathscr{D}(\mathfrak{G})\right]^{\operatorname{det}^{m}}$. The proof will need to be a little more involved since the radial component map $\mathfrak{R}$ from (4.2.2) actually induces the zero map on $\mathscr{D}(\mathfrak{G})^{\text {det }}$. This can presumably be circumvented by using a "Fourier transform" of $\Re$. We will, however, take an alternative approach by showing in the next lemma that there is an easy direct way to move between the Q's and P's that makes such a result unnecessary.

5.6. By [De, Remark 2.2] there is an isomorphism $\phi_{c}: \mathrm{H}_{c} \rightarrow \mathrm{H}_{-c}$ defined by $\phi_{c}(x)=x$, $\phi_{c}\left(D_{c}(y)\right)=D_{-c}(y)$ and $\phi_{c}(w)=\operatorname{sign}(w) w$, for $x \in \mathbb{C}[\mathfrak{h}], y \in \mathbb{C}\left[\mathfrak{h}^{*}\right]$ and $w \in W$. Note that $\phi_{c}(e)=e_{-}$in the notation from (2.3) . Since $\phi_{c}(\delta)=\delta$ the map $\phi_{c}$ extends to an automorphism of $\mathrm{H}^{\mathrm{reg}}=\mathrm{H}_{c}\left[\delta^{-1}\right]$ which will still be written $\phi_{c}$. The reader should be warned that the action of $\phi_{c}$ on $\mathrm{H}^{\text {reg }}$ does depend upon $c$.

Lemma. Fix $c \in \mathbb{C}$. Then:

(1) $\phi_{c+1}(z)=\delta^{-2} \phi_{c}(z) \delta^{2}$ for all $z \in \mathrm{U}^{\mathrm{reg}}$.

(2) $\phi_{c+1}\left(\mathrm{U}_{c}\right)=\delta^{-1} \mathrm{U}_{-c-1} \delta$.

(3) $\phi_{c+1}\left({ }_{c+j} \mathrm{P}_{c}\right)=\delta^{2 j-1}\left({ }_{-c-j-1} \mathrm{Q}_{-c-1}\right) \delta$ for all $j>0$.

Proof. (1) By [EG, Proposition 4.9 and (11.33)], $\mathbf{U}_{c}$ is generated as an algebra by $\mathbb{C}[\mathfrak{h}]^{W}$ and $\nabla_{c}^{2}=\sum_{i=1}^{n} D_{c}\left(y_{i}\right)^{2}$; thus $\mathscr{D}\left(\mathfrak{h}^{\text {reg }}\right)^{W}$ is generated by $\mathbb{C}\left[\mathfrak{h}^{\text {reg }}\right]^{W}$ and $\nabla_{c}^{2}$. Thus we only need to confirm (1) for elements $p e \in \mathbb{C}\left[\mathfrak{h}^{\text {reg }}\right]^{W} e$ and for $\nabla_{c}^{2} e$. The former is obvious since

$$
\delta^{-2} \phi_{c}(p e) \delta^{2}=\delta^{-2} p e_{-} \delta^{2}=p e_{-}=\phi_{c+1}(p e) \quad \text { for } p \in \mathbb{C}\left[\mathfrak{h}^{\text {reg }}\right]^{W} .
$$

By [He, Theorem 3.1] we have $\delta^{-1} \nabla_{c+1}^{2} e_{-} \delta=\nabla_{c}^{2} e$ for all $c \in \mathbb{C}$ (note that our scalar $c$ is the scalar $-k$ in [He] $)$. Thus, (1) follows from the chain of equalities

$$
\delta^{-2} \phi_{c}\left(\nabla_{c}^{2} e\right) \delta^{2}=\delta^{-2} \nabla_{-c}^{2} e_{-} \delta^{2}=\delta^{-1} \nabla_{-c-1}^{2} e \delta=\phi_{c+1}\left(\delta^{-1} \nabla_{c+1}^{2} e_{-} \delta\right)=\phi_{c+1}\left(\nabla_{c}^{2} e\right) .
$$

(2) This is equivalent to the assertion that $\phi_{-c-1}\left(\mathrm{U}_{-c-1}\right)=\delta \mathrm{U}_{c} \delta^{-1}$. By [EG, Proposition 4.9 and (11.33)], again, it is enough to confirm this for $\mathbb{C}[\mathfrak{h}]^{W} e$ and $\nabla_{-c-1}^{2} e$. This is trivial for $\mathbb{C}[\mathfrak{h}]^{W} e$ while $\left[\mathrm{He}\right.$, Theorem 3.1] gives $\phi_{-c-1}\left(\nabla_{-c-1}^{2} e\right)=\nabla_{c+1}^{2} e_{-}=\delta \nabla_{c}^{2} e \delta^{-1}$, as required. 
(3) For $j=1$ we have

$$
\phi_{c+1}\left({ }_{c+1} \mathrm{P}_{c}\right)=\phi_{c+1}\left(e \mathrm{H}_{c+1} \delta e\right)=e_{-} \mathrm{H}_{-c-1} \delta e_{-}=\delta e \delta^{-1} \mathrm{H}_{-c-1} e \delta=\delta\left({ }_{-c-2} \mathrm{Q}_{-c-1}\right) \delta,
$$

as desired. Now assume that the result holds for some $j \geq 1$. By part $(1), \phi_{c+j+1}(z)=$ $\delta^{-2 j} \phi_{c+1}(z) \delta^{2 j}$ for $z \in \mathrm{U}^{\mathrm{reg}}$. Applying the case $j=1$ gives

$$
\delta\left({ }_{-c-j-2} \mathrm{Q}_{-c-j-1}\right) \delta=\phi_{c+j+1}\left({ }_{c+j+1} \mathrm{P}_{c+j}\right)=\delta^{-2 j}\left(\phi_{c+1}\left({ }_{c+j+1} \mathrm{P}_{c+j}\right)\right) \delta^{2 j} .
$$

Equivalently

$$
\phi_{c+1}\left(c+j+1 \mathrm{P}_{c+j}\right)=\delta^{2 j+1}\left(-c-j-2 \mathrm{Q}_{-c-j-1}\right) \delta^{1-2 j} .
$$

To complete the proof, by induction, we obtain

$$
\begin{aligned}
\phi_{c+1}\left({ }_{c+j+1} \mathrm{P}_{c}\right) & =\phi_{c+1}\left({ }_{c+j+1} \mathrm{P}_{c+j}\right) \phi_{c+1}\left({ }_{c+j} \mathrm{P}_{c}\right) \\
& =\delta^{2 j+1}\left({ }_{-c-j-2} \mathrm{Q}_{-c-j-1}\right) \delta^{1-2 j} \cdot \delta^{2 j-1}\left({ }_{-c-j-1} \mathrm{Q}_{-c-1}\right) \delta \\
& =\delta^{2 j+1}\left({ }_{-c-j-2} \mathrm{Q}_{-c-1}\right) \delta .
\end{aligned}
$$

5.7. Completion of the proof of Theorem 1.7. By Remark 5.5 it only remains to prove Theorem 1.7(2). Changing notation slightly, we consider ${ }_{d+m} \mathrm{P}_{d}$ where $d \in \mathbb{C}$ is chosen so that the numbers $d+1, d+2, \ldots, d+m-1$ are all good. Thus we need to prove that $\operatorname{gr}_{d+m} \mathrm{P}_{d}=A^{m} \delta^{m} e$.

Consider $\phi_{d+1}$. By construction, this morphism preserves the differential operator filtration on $\mathrm{H}^{\mathrm{reg}}$ and gr $\phi_{d+1}$ is then the automorphism that is the identity on $\mathbb{C}\left[\mathfrak{h} \times \mathfrak{h}^{*}\right]$ and sends $w \in W$ to $\operatorname{sign}(w) w$. Moreover, if $c=-d-1$ then $c-1, c-2, \ldots, c-m+1$ are also good. Thus Lemma 5.6 and Theorem 5.3 can be applied to show that

$$
\begin{aligned}
\left(\operatorname{gr} \phi_{d+1}\right)\left(\operatorname{gr}_{d+m} \mathrm{P}_{d}\right) & =\operatorname{gr}\left(\phi_{d+1}\left({ }_{d+m} \mathrm{P}_{d}\right)\right)=\operatorname{gr}\left(\delta^{2 m-1}\left({ }_{-d-m-1} \mathrm{Q}_{-d-1}\right) \delta\right) \\
& =\operatorname{gr}\left(\delta^{2 m-1}\left(_{c-m} \mathrm{Q}_{c}\right) \delta\right)=\delta^{2 m-1}\left(\delta^{-m} A^{m} e\right) \delta=\delta^{m-1} A^{m} \delta e_{-}
\end{aligned}
$$

and hence $\operatorname{gr}\left({ }_{d+m} \mathrm{P}_{d}\right)=\operatorname{gr} \phi_{d+1}^{-1}\left(\delta^{m-1} A^{m} \delta e_{-}\right)=\delta^{m} A^{m} e$, as required.

5.8. We finish this section by making explicit the connection between shifting by Q's, by P's and applying the various morphisms $\phi_{c}$ 's of Section [5.6. The equality $\phi_{c+1}\left(\mathrm{U}_{c}\right)=$ $\delta^{-1} U_{-c-1} \delta$ from Lemma 5.6(2) induces an equivalence of categories

$$
\Omega_{c}: \mathrm{U}_{c^{-}} \bmod \stackrel{\sim}{\longrightarrow} \mathrm{U}_{-c-1}-\bmod , \quad V \mapsto V,
$$

where $V \in \mathrm{U}_{c^{-}}$mod becomes a $\mathrm{U}_{-c-1}$-module via $z * v=\phi_{-c-1}\left(\delta^{-1} z \delta\right) v$ for $z \in \mathrm{U}_{-c-1}$ and $v \in V$.

Proposition. Let $c \in \mathbb{C}$ and set $d=-c-1$. For any integer $m \geq 0$ there is an isomorphism of functors ${ }_{d+m} \mathrm{P}_{d} \otimes \mathrm{U}_{d} \Omega_{c}(-) \cong \Omega_{c-m} \circ\left({ }_{c-m} \mathrm{Q}_{c} \otimes \mathrm{U}_{c}(-)\right)$ that makes the 
following diagram commute

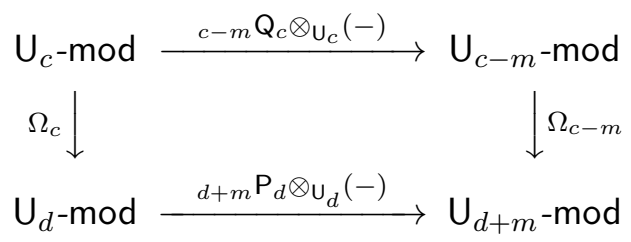

Proof. Let $V$ be a $\mathrm{U}_{c}$-module. By Lemma [5.6(3) ${ }_{c-m} \mathrm{Q}_{c}=\delta^{1-2 m} \phi_{-c}\left(d+m \mathrm{P}_{d}\right) \delta^{-1}$, so we are asserting the existence a natural isomorphism between ${ }_{d+m} \mathrm{P}_{d} \otimes \mathrm{U}_{d} V$ (with $\mathrm{U}_{d}$-action on $V$ induced from $\Omega_{c}$ ) and $\delta^{1-2 m} \phi_{-c}\left(d+m \mathrm{P}_{d}\right) \delta^{-1} \otimes \mathrm{U}_{c} V$ (with $\mathrm{U}_{d+m}$-action induced from $\left.\Omega_{c-m}\right)$. The only choice is the mapping $p \otimes v \mapsto \delta^{1-2 m} \phi_{-c}(p) \delta^{-1} \otimes \mathbf{U}_{c} v$ for $p \in{ }_{d+m} \mathrm{P}_{d}$ and $v \in V$. We need to check that this is well-defined and that it is a $\mathrm{U}_{d+m}$-module homomorphism.

Pick $z \in \mathrm{U}_{d}, p \in{ }_{d+m} \mathrm{P}_{d}$ and $v \in V$. For well-definedness we have

$$
\begin{aligned}
p z \otimes v-p \otimes z * v & \mapsto \delta^{1-2 m}\left(\phi_{-c}(p z) \delta^{-1} \otimes \mathbf{U}_{c} v-\phi_{-c}(p) \delta^{-1} \otimes \mathbf{U}_{c} z * v\right) \\
& =\delta^{1-2 m} \phi_{-c}(p)\left(\phi_{-c}(z) \delta^{-1} \otimes \mathbf{U}_{c} v-\delta^{-1} \otimes \mathbf{U}_{c} \phi_{-c-1}\left(\delta^{-1} z \delta\right) v\right) \\
& =\delta^{1-2 m} \phi_{-c}(p)\left(\delta^{-2} \phi_{-c-1}(z) \delta \otimes \mathbf{U}_{c} v-\delta^{-1} \otimes \mathbf{U}_{c} \phi_{-c-1}\left(\delta^{-1} z \delta\right) v\right)=0,
\end{aligned}
$$

where in the first equality of the last line we used Lemma 5.6(1).

Now let $z \in \mathrm{U}_{d+m}$. Using Lemma 5.6(1) and induction we have

$$
\begin{aligned}
z p \otimes v & \mapsto \delta^{1-2 m} \phi_{-c}(z p) \delta^{-1} \otimes \mathbf{U}_{c} v \\
& =\delta^{-2(m-1)} \phi_{-c}\left(\delta^{-1} z \delta\right) \delta^{2(m-1)} \delta^{1-2 m} \phi_{-c}(p) \delta^{-1} \otimes \mathbf{U}_{c} v \\
& =\phi_{-c+m-1}\left(\delta^{-1} z \delta\right) \delta^{1-2 m} \phi_{-c}(p) \delta^{-1} \otimes \mathbf{U}_{c} v \\
& =z *\left(\delta^{1-2 m} \phi_{-c}(p) \delta^{-1} \otimes_{\mathbf{U}_{c}} v\right) .
\end{aligned}
$$

This confirms $\mathrm{U}_{d+m}$-equivariance.

\section{Shift functors for $\mathscr{D}$-modules and Cherednik algebras}

6.1. The morphism ${ }_{c+m} \mathrm{P}_{c} \otimes-: \mathrm{U}_{c}-\bmod \rightarrow \mathrm{U}_{c+m}$-mod is fundamental to the representation theory of $\mathrm{U}_{c}$, as is illustrated by much of [GS2]. There is a similar translation functor for twisted $\mathscr{D}$-modules on projective space given by tensoring with $\mathcal{O}(m n)$ (see Section 6.5 for the precise definition). In this section we show that these functors are naturally intertwined by hamiltonian reduction, thereby proving Theorem 1.9 from the introduction. Before stating the result we will need some definitions.

6.2. Projectivisation. The quantum hamiltonian reduction of Theorem 2.8 can be performed in two steps: first with respect to the subgroup of scalar matrices $\mathbb{C}^{\times} \subset$ $G=G L(V)$; and then with respect to the subgroup $S L(V) \subset G$. Recall that $G$ acts diagonally on $\mathfrak{G}=\mathfrak{g} \times V$ and so $\mathbb{C}^{\times}$acts trivially on $\mathfrak{g}$ and by dilations $\lambda \circ v=\lambda v$ on 
$V=\mathbb{C}^{n}$. As in (2.6), the identity matrix in $\mathfrak{g l}(V)$ is written 1 . Put

$$
V^{\circ}=V \backslash\{0\} ; \quad \mathbb{P}=\mathbb{P}(V)=V^{\circ} / \mathbb{C}^{\times} ; \quad \mathfrak{G}^{\circ}=\mathfrak{g} \times V^{\circ} ; \quad \text { and } \quad \mathfrak{X}=\mathfrak{g} \times \mathbb{P} .
$$

Lemma. Let $c \in \mathbb{C}$ and assume that if $n=2$ then $2 c \notin \mathbb{Z}_{\leq 0}$. Then restriction provides a natural isomorphism

$$
\chi: \mathscr{D}(\mathfrak{G}) / \mathscr{D}(\mathfrak{G}) \tau(\mathbf{1}-n c) \stackrel{\sim}{\longrightarrow} \Gamma\left(\mathfrak{G}^{\circ}, \mathscr{D}_{\mathfrak{G}} \circ / \mathscr{D}_{\mathfrak{G}} \circ(\mathbf{1}-n c)\right) .
$$

Proof. Without loss of generality we need only consider $V$ instead of $\mathfrak{G}$.

Set $v=\tau(\mathbf{1}-n c)$. Multiplication by $v$ on the right yields a short exact sequence of sheaves on $V$ :

$$
0 \rightarrow \mathscr{D}_{V} \rightarrow \mathscr{D}_{V} \rightarrow \mathscr{D}_{V} / \mathscr{D}_{V} v \rightarrow 0 .
$$

Hence on $V^{\circ}$ we obtain the long exact sequence

$$
\begin{aligned}
& 0 \longrightarrow \Gamma\left(V^{\circ}, \mathscr{D}_{V}\right) \stackrel{\times v}{\longrightarrow} \quad \Gamma\left(V^{\circ}, \mathscr{D}_{V}\right) \longrightarrow \Gamma\left(V^{\circ}, \mathscr{D}_{V} / \mathscr{D}_{V} v\right) \longrightarrow \\
& \longrightarrow H^{1}\left(V^{\circ}, \mathscr{D}_{V}\right) \stackrel{\times v}{\longrightarrow} H^{1}\left(V^{\circ}, \mathscr{D}_{V}\right) \longrightarrow \ldots
\end{aligned}
$$

Assume first that $n \geq 3$. Then $H^{1}\left(V^{\circ}, \mathcal{O}_{V}\right) \cong H_{\{0\}}^{2}\left(V, \mathcal{O}_{V}\right)=0$ since $H_{\{0\}}^{i}\left(V, \mathcal{O}_{V}\right)=0$ for all $i<n=\operatorname{dim} V$. Since $\mathscr{D}_{V}$ is free as a sheaf of $\mathcal{O}_{V}$-modules, it follows that $H^{1}\left(V^{\circ}, \mathscr{D}_{V}\right)=0$. Since $\mathbb{C}[V]$ is just a polynomial ring and $\operatorname{dim} V \backslash V^{\circ} \leq \operatorname{dim} V-2$, we have $\mathbb{C}\left[V^{\circ}\right]=\Gamma\left(V^{\circ}, \mathcal{O}_{V^{\circ}}\right)=\mathbb{C}[V]$ by Hartog's theorem. By freeness, $\Gamma\left(V^{\circ}, \mathscr{D}_{V^{\circ}}\right)=\mathscr{D}(V)$ and so we are done if $n \geq 3$.

Now assume that $n=2$ and write $\mathbb{C}[V]=\mathbb{C}[x, y]$. A simple calculation in Čech cohomology using the open sets $D(x)=V \backslash\{x=0\}$ and $D(y)=V \backslash\{y=0\}$ shows that

$$
H^{1}\left(V^{\circ}, \mathcal{O}_{V}\right) \cong \frac{\mathbb{C}\left[x^{ \pm 1}, y^{ \pm 1}\right]}{\left(\mathbb{C}\left[x^{ \pm 1}, y\right]+\mathbb{C}\left[x, y^{ \pm 1}\right]\right)}
$$

Denote this space by $S$, so that $H^{1}\left(V^{\circ}, \mathscr{D}_{V}\right)=\bigoplus_{i, j \geq 0} S \partial_{x}^{i} \partial_{y}^{j}$, where $\partial_{x}=\partial / \partial x$, etc.

We claim that the mapping $H^{1}\left(V^{\circ}, \mathscr{D}_{V}\right) \stackrel{\times v}{\rightarrow} H^{1}\left(V^{\circ}, \mathscr{D}_{V}\right)$ is injective if $2 c \notin \mathbb{Z}_{\geq 0}$. To see this let

$$
0 \neq z=\sum_{i, j \geq 0} s_{i, j} \partial_{x}^{i} \partial_{y}^{j} \in H^{1}\left(V^{\circ}, \mathscr{D}_{V}\right)
$$

for some $s_{i, j} \in S$. By (2.7) we have $\tau(\mathbf{1})=-x \partial_{x}-y \partial_{y}$ and so

$$
\begin{aligned}
z v=z \tau(\mathbf{1}-2 c) & =\sum_{i, j \geq 0}\left(s_{i, j} \tau(\mathbf{1})-(i+j+2 c) s_{i, j}\right) \partial_{x}^{i} \partial_{y}^{j} \\
& =\sum_{i, j \geq 0}\left(s_{i-1, j} x+s_{i, j-1} y-(i+j+2 c) s_{i, j}\right) \partial_{x}^{i} \partial_{y}^{j} .
\end{aligned}
$$

Thus if $z v=0$ then $s_{i-1, j} x+s_{i, j-1} y-(i+j+2 c) s_{i, j}=0$ for all $i, j \geq 0$. But consideration of the lowest degree $\left(i_{0}, j_{0}\right)$ of nonzero monomials in $z$ then shows that $i_{0}+j_{0}+2 c=0$, contradicting the hypothesis that $2 c \notin \mathbb{Z}_{\leq 0}$. It follows that the morphism

$$
\Gamma\left(V, \mathscr{D}_{V}\right) \cong \Gamma\left(V^{\circ}, \mathscr{D}_{V}\right) \rightarrow \Gamma\left(V^{\circ}, \mathscr{D}_{V} / \mathscr{D}_{V} v\right)
$$

is surjective, as required. 
Remark. The lemma fails when $n=2$ and $2 c \in \mathbb{Z}_{\leq 0}$. In the notation of the above proof we have the following equality in $\mathscr{D}_{V}(D(x y))$ :

$$
x^{-1} y^{-1} \partial_{x}^{-2 c} v=-x^{-1} \partial_{x}^{-2 c} \partial_{y}-y^{-1} \partial_{x}^{-2 c+1} .
$$

Thus $x^{-1} \partial_{x}^{-2 c} \partial_{y}=-y^{-1} \partial_{x}^{-2 c+1}$ in $\left(\mathscr{D}_{V} / \mathscr{D}_{V} v\right)(D(x y))$. It follows that the sections $x^{-1} \partial_{x}^{-2 c} \partial_{y} \in\left(\mathscr{D}_{V} / \mathscr{D}_{V} v\right)(D(x))$ and $-y^{-1} \partial_{x}^{-2 c+1} \in\left(\mathscr{D}_{V} / \mathscr{D}_{V} v\right)(D(y))$ extend to a section of $\left(\mathscr{D}_{V} / \mathscr{D}_{V} v\right)\left(V^{\circ}\right)$ which is not in the image of $\chi$.

6.3. Following [GG, Section 5.1], for each $c \in \mathbb{C}$ we introduce an algebra

$$
\mathscr{D}_{c}(\mathfrak{X}) \stackrel{\text { def }}{=}\left(\frac{\mathscr{D}(\mathfrak{G})}{\mathscr{D}(\mathfrak{G}) \tau(\mathbf{1}-n c)}\right)^{\mathbb{C}^{\times}} .
$$

The algebra on the right hand side of (6.3.1) is a quantum hamiltonian reduction with respect to the group $\mathbb{C}^{\times}$at the point $c$.

Using Theorem 2.8, we can apply the formalism of hamiltonian reduction, as outlined in [GG, Section 7], to $\mathscr{D}_{c+1}(\mathfrak{X})$ and $\mathrm{U}_{c}$. Let $\left(\mathscr{D}_{c+1}(\mathfrak{X}), S L(V)\right)$-mod denote the category whose objects are the finitely generated $S L(V)$-equivariant $\mathscr{D}_{c+1}(\mathfrak{X})$-modules on which the action of $\mathfrak{s l}(V)$ induced from the $S L(V)$-equivariance agrees with the action of $\mathfrak{s l}(V)$ induced from the homomorphism $\mathfrak{s l}(V) \stackrel{\tau}{\longrightarrow} \mathscr{D}(\mathfrak{G})^{\mathbb{C}^{\times}} \longrightarrow \mathscr{D}_{c+1}(\mathfrak{X})$. We have the following functor of hamiltonian reduction:

$$
\mathbb{H}_{c}:\left(\mathscr{D}_{c+1}(\mathfrak{X}), S L(V)\right) \text {-mod } \longrightarrow \mathrm{U}_{c^{-}} \bmod ; \quad \mathcal{F} \mapsto \mathbb{H}_{c}(\mathcal{F})=\mathcal{F}^{S L(V)} .
$$

Since $S L(V)$ is reductive, the functor $\mathbb{H}_{c}$ is exact and, by [GG, Proposition 7.1], it has a left adjoint

$$
\begin{aligned}
{ }^{\top} \mathbb{H}_{c}: \mathrm{U}_{c}-\bmod & \longrightarrow\left(\mathscr{D}_{c+1}(\mathfrak{X}), S L(V)\right) \text {-mod; } \\
M & \longmapsto\left(\mathscr{D}_{c+1}(\mathfrak{X}) / \mathscr{D}_{c+1}(\mathfrak{X}) \tau\left(I_{c+1}\right)\right) \otimes \mathrm{U}_{c} M .
\end{aligned}
$$

6.4. Next, let $\mathscr{D}_{\mathfrak{X}, c}$ denote the sheaf of $(-n c)$-twisted differential operators on $\mathfrak{X}$. It follows from [GG, Equation 5.1] that $\mathscr{D}_{\mathfrak{X}, c}$ has global sections $\Gamma\left(\mathfrak{X}, \mathscr{D}_{\mathfrak{X}, c}\right)=\mathscr{D}_{c}(\mathfrak{X})$. For any $m \in \mathbb{Z}$, let $\mathcal{O}(m)$ be the pull-back of the standard line bundle $\mathcal{O}_{\mathbb{P}}(m)$ via the projection $\mathfrak{X}=\mathfrak{g} \times \mathbb{P} \rightarrow \mathbb{P}$. Tensoring with $\mathcal{O}(n m)$ yields a functor

$$
\mathscr{D}_{\mathfrak{X}, c}-\bmod \longrightarrow \mathscr{D}_{\mathfrak{X}, c-m}-\bmod , \quad \mathcal{F} \mapsto \mathcal{O}(n m) \otimes_{\mathcal{O}_{\mathfrak{X}}} \mathcal{F}
$$

6.5. Assume now that $n(c+1) \in \mathbb{C} \backslash \mathbb{Z}_{>0}$. As in [GG, Proposition 5.4], we can apply the Beilinson-Bernstein theorem to give an equivalence of categories

$$
\Gamma(\mathfrak{X},-): \mathscr{D}_{\mathfrak{X}, c+1} \text {-mod } \stackrel{\sim}{\longrightarrow} \mathscr{D}_{c+1}(\mathfrak{X}) \text {-mod, } \quad \mathcal{F} \mapsto \Gamma(\mathfrak{X}, \mathcal{F}) .
$$

We write

$$
\mathbb{S}^{m}: \mathscr{D}_{c+1}(\mathfrak{X})-\bmod \longrightarrow \mathscr{D}_{c-m+1}(\mathfrak{X})-\bmod , \quad F \mapsto F(n m)
$$

for the functor that corresponds to the functor (6.4.1) via the Beilinson-Bernstein equivalence. 
Theorem. Fix $c \in \mathbb{C}$ and a positive integer $m$ such that each of the numbers $c-1, c-2$, $\ldots, c-m+1$ is good and $n(c+1) \notin \mathbb{Z}_{>0}$ (respectively, $n(c+1) \notin \mathbb{Z}$ if $\left.n=2\right)$. Then there is an isomorphism of functors $\mathbb{H}_{c-m} \circ \mathbb{S}^{m} \circ{ }^{\top} \mathbb{H}_{c}(-) \cong{ }_{c-m} Q_{c} \otimes \mathrm{U}_{c}(-)$ that makes the following diagram commute

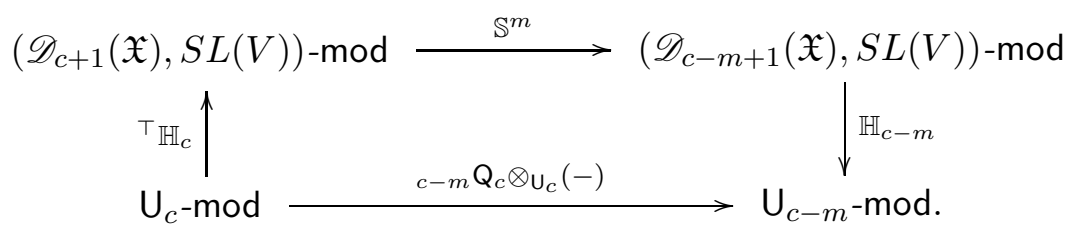

6.6. Before proving the theorem we note that, by Proposition 5.8 , it has the following equivalent formulation in terms of the P's. For $d \in \mathbb{C}$ we set

$$
\widetilde{\mathbb{H}}_{d} \stackrel{\text { def }}{=} \Omega_{-d-1} \circ \mathbb{H}_{-d-1}:\left(\mathscr{D}_{-d}(\mathfrak{X}), S L(V)\right)-\bmod \longrightarrow \mathrm{U}_{d^{-}} \text {mod, }
$$

where $\Omega_{d}$ is defined in (5.8). This has left adjoint

$$
{ }^{\top} \widetilde{\mathbb{H}}_{d}={ }^{\top} \mathbb{H}_{-d-1} \circ \Omega_{-d-1}^{-1}={ }^{\top} \mathbb{H}_{-d-1} \circ \Omega_{d} .
$$

Then, using the fact that $d$ is good if and only if $-d-1$ is good, we obtain:

Corollary. Fix $c \in \mathbb{C}$ and a positive integer $m$ such that $c+1, \ldots, c+m-1$ are all good and that $n c \notin \mathbb{Z}_{<0}$ (respectively $n c \notin \mathbb{Z}$ if $n=2$ ). Then there is a commutative diagram:

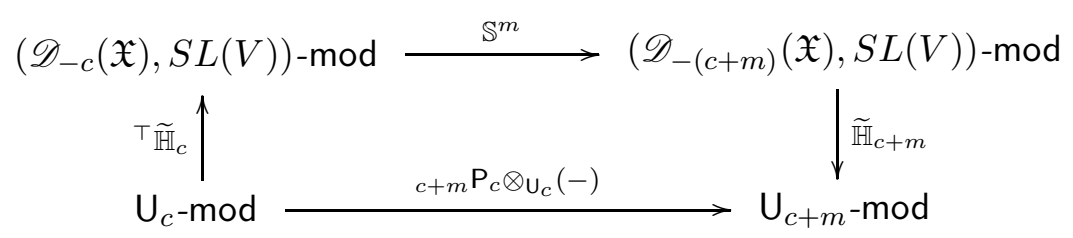

Remark. Theorem 1.9 is a special case of this corollary.

6.7. Recall that $\mathbb{C}^{\times} \subset G L(V)$ is the central subgroup consisting of multiples of the identity matrix Id. Given a $G L(V)$-representation $E$ we will denote by $E^{(m)}$ the set of semi-invariants $\left\{e \in E:(z \mathrm{Id}) \cdot e=z^{m n} e\right.$ for all $\left.z \in \mathbb{C}^{\times}\right\}$. For $d \in \mathbb{C}$, we define

$$
{ }_{d-m} \mathfrak{D}_{d} \stackrel{\text { def }}{=}\left[\frac{\mathscr{D}(\mathfrak{G})}{\mathscr{D}(\mathfrak{G}) \tau(\mathbf{1}-n d)}\right]^{(-m)} \quad \text { for } m \in \mathbb{Z} .
$$

Note that $N^{\operatorname{det}^{-m}}=\left(N^{S L}\right)^{(-m)}$ for any $G L(V)$-module $N$. By Lemma $2.2,{ }_{d-m} \mathfrak{D}_{d}$ has a natural $\left(\mathscr{D}_{d-m}(\mathfrak{X}), \mathscr{D}_{d}(\mathfrak{X})\right)$-bimodule structure and it also has the following useful properties.

Lemma. (1) Fix $c \in \mathbb{C}$ and a positive integer $m$ such that $n(c+1) \notin \mathbb{Z}_{>0}$ (respectively $n(c+1) \notin \mathbb{Z}$ if $n=2)$. Then, for any $F \in\left(\mathscr{D}_{c+1}(\mathfrak{X}), S L(V)\right)$-mod,

$$
\mathbb{S}^{m}(F)=\Gamma\left(\mathfrak{X}, \mathcal{O}(n m) \otimes_{\mathcal{O}_{\mathfrak{X}}} \mathscr{D}_{\mathfrak{X}, c+1}\right) \otimes_{\mathscr{D}_{c+1}(\mathfrak{X})} F \cong{ }_{c-m+1} \mathfrak{D}_{c+1} \otimes_{\mathscr{D}_{c+1}(\mathfrak{X})} F
$$


(2) Assume that $c-j$ is good for $1 \leq j \leq m-1$. There are isomorphisms of $\left(\mathrm{U}_{c-m}, \mathrm{U}_{c}\right)$ bimodules

$$
\left(\frac{{ }_{c-m+1} \mathfrak{D}_{c+1}}{{ }_{c-m+1} \mathfrak{D}_{c+1} \tau\left(I_{c+1}\right)}\right)^{S L} \cong\left(\frac{\mathscr{D}(\mathfrak{G})}{\mathscr{D}(\mathfrak{G}) \tau\left(I_{c+1}\right)}\right)^{\operatorname{det}^{-m}} \cong{ }_{c-m} \mathrm{Q}_{c} .
$$

Proof. (1) The first equality in (6.7.1) follows from the Beilinson-Bernstein equivalence (6.5.1) and the definition of $\mathbb{S}^{m}$. For the displayed isomorphism, we consider the principal $\mathbb{C}^{\times}$-bundle $p: V^{\circ} \longrightarrow \mathbb{P}$. Then by equivariant descent (see [SGA1, Chapter VII, Section 1]) we have

$$
\begin{aligned}
\mathcal{O}_{\mathbb{P}}(m n) \otimes_{\mathcal{O}_{\mathbb{P}}} \mathscr{D}_{\mathbb{P}, c+1} & \cong p_{*}\left(\left(\mathcal{O}_{V^{\circ}} \otimes_{\mathbb{C}} \operatorname{det}^{m}\right) \otimes_{\mathcal{O}_{V}} \frac{\mathscr{D}_{V^{\circ}}}{\mathscr{D}_{V} \tau(\mathbf{1}-n(c+1))}\right)^{\mathbb{C}^{\times}} \\
& \cong p_{*}\left(\frac{\mathscr{D}_{V^{\circ}}}{\mathscr{D}_{V^{\circ} \tau(\mathbf{1}-n(c+1))}}\right)^{(-m)} .
\end{aligned}
$$

It follows that

$$
\begin{aligned}
\Gamma\left(\mathbb{P}, \mathcal{O}_{\mathbb{P}}(n m) \otimes_{\mathbb{P}} \mathscr{D}_{\mathbb{P}, c+1}\right) & \cong \Gamma\left(\mathbb{P}, p_{*}\left(\frac{\mathscr{D}_{V^{\circ}}}{\mathscr{D}_{V} \circ(\mathbf{1}-n(c+1))}\right)\right)^{(-m)} \\
& \cong \Gamma\left(V^{\circ}, \frac{\mathscr{D}_{V^{\circ}}}{\mathscr{D}_{V} \tau(\mathbf{1}-n(c+1))}\right)^{(-m)}
\end{aligned}
$$

Now $\mathscr{D}_{\mathfrak{G}^{\circ}}=\mathscr{D}_{\mathfrak{g}} \otimes \mathscr{D}_{V^{\circ}}$, with $\mathbb{C}^{\times}$acting trivially on $\mathscr{D}_{\mathfrak{g}}$. Therefore, combining (6.2.1) with (6.7.2) gives the following isomorphism of $\left(\mathscr{D}_{c-m+1}(\mathfrak{X}), \mathscr{D}_{c+1}(\mathfrak{X})\right)$-bimodules:

$$
\begin{aligned}
{ }_{c-m+1} \mathfrak{D}_{c+1} & \cong\left(\frac{\mathscr{D}(\mathfrak{G})}{\mathscr{D}(\mathfrak{G}) \tau(\mathbf{1}-n(c+1))}\right)^{(-m)} \cong \Gamma\left(\mathfrak{G}^{\circ}, \frac{\mathscr{D}_{\mathfrak{G} \circ}}{\mathscr{D}_{\mathfrak{G}^{\circ} \tau(\mathbf{1}-n(c+1))}}\right)^{(-m)} \\
& \cong \Gamma\left(\mathbb{P}, \mathcal{O}_{\mathbb{P}}(n m) \otimes_{\mathbb{P}} \mathscr{D}_{\mathbb{P}, c+1}\right) \otimes \mathscr{D}(\mathfrak{g}) \cong \Gamma\left(\mathfrak{X}, \mathcal{O}(n m) \otimes_{\mathcal{O}_{\mathfrak{X}}} \mathscr{D}_{\mathfrak{X}, c+1}\right),
\end{aligned}
$$

as required.

(2) As a morphism of $\mathscr{D}(\mathfrak{G})^{S L}$-modules, the first isomorphism follows from the observation that $N^{\mathrm{det}^{-m}}=\left(N^{S L}\right)^{(-m)}$ for any $G L(V)$-module $N$. By Lemma 2.2 this restricts to give an isomorphism of $\left(\mathscr{D}_{c-m+1}(\mathfrak{X}), \mathscr{D}_{c+1}(\mathfrak{X})\right)$-bimodules; equivalently of $\left(\mathbf{U}_{c-m}, \mathbf{U}_{c}\right)$-bimodules. The second isomorphism is just Theorem 1.6.

6.8. Proof of Theorem 6.5. Let $M \in \mathrm{U}_{c^{-}}$mod. By Lemma 6.7(1)

$$
\begin{aligned}
& \mathbb{S}^{m} \circ{ }^{\top} \mathbb{H}_{c}(M)={ }_{c-m+1} \mathfrak{D}_{c+1} \otimes_{\mathscr{D}_{c+1}(\mathfrak{X})}\left(\frac{\mathscr{D}_{c+1}(\mathfrak{X})}{\mathscr{D}_{c+1}(\mathfrak{X}) \tau\left(I_{c+1}\right)} \otimes_{\mathrm{U}_{c}} M\right) \\
& \cong\left(\frac{c-m+1 \mathfrak{D}_{c+1}}{c+m-1 \mathfrak{D}_{c+1} \tau\left(I_{c+1}\right)}\right) \otimes_{\mathbf{U}_{c}} M . \\
& 26
\end{aligned}
$$


Thus Lemma 6.7(2) implies that

$$
\begin{gathered}
\mathbb{H}_{c-m} \circ \mathbb{S}^{m} \circ{ }^{\top} \mathbb{H}_{c}(M) \cong\left(\left(\frac{c-m+1}{\mathfrak{D}_{c+1}}\right) \otimes_{\mathrm{U}_{c}} M\right)^{S L} \\
=\left(\frac{c-m+1 \mathfrak{D}_{c+1} \tau\left(I_{c+1}\right)}{{ }_{c+m-1} \mathfrak{D}_{c+1} \tau\left(I_{c+1}\right)}\right)^{S L} \otimes \mathrm{U}_{c} M \cong{ }_{c-m} \mathrm{Q}_{c} \otimes M .
\end{gathered}
$$

\section{Characteristic CyCles}

7.1. Let $M$ be a filtered, finitely generated $\mathrm{U}_{c}$-module. In GS2 the authors used the $\mathbb{Z}$-algebra associated to the modules $\left\{{ }_{c+a} \mathrm{P}_{c}\right\}$ to construct a characteristic cycle $\operatorname{ch}(M)$ inside the Hilbert scheme Hilb ${ }^{n} \mathbb{C}^{2}$ that then proved useful in studying the representation theory of $\mathrm{U}_{c}$. Using quantum hamiltonian reduction, the authors of [GG] define a second such characteristic cycle. This leads to the natural question of whether these varieties are equal; see [GG, (7.17)]. In this section we show that this is indeed the case.

7.2. Hilbert schemes. We write $\operatorname{Coh}(X)$ for the category of coherent sheaves on a scheme $X$. If $B=\bigoplus_{m \geq 0} B_{m}$ is a finitely generated graded commutative algebra let $B$-grmod denote the category finitely generated graded left $B$-modules and write $\mathbb{F}(M)$ for the coherent sheaf on the scheme Proj $B$ corresponding to the module $M \in B$-grmod.

Set $A=\bigoplus_{m \geq 0} A^{m}$, where the $A^{m}$ are defined as in (4.3); thus [Ha2, Proposition 2.6] implies that $\operatorname{Proj}(A) \cong \operatorname{Hilb}^{n} \mathbb{C}^{2}$, the Hilbert scheme of $n$ points in $\mathbb{C}^{2}$. Following Ha2] and $\left[\mathrm{Na}\right.$, there is the following diagram of schemes over $\left(\mathfrak{h} \times \mathfrak{h}^{*}\right) / W$ :

$$
\mathcal{M} \stackrel{j}{\longleftarrow} \mathcal{M}^{\text {cyc }} \stackrel{p}{\longrightarrow} \operatorname{Hilb}^{n} \mathbb{C}^{2} \cong \operatorname{Proj} A \cong \operatorname{Proj}\left(\bigoplus_{m \geq 0} \mathbb{C}[\mathcal{M}]^{\operatorname{det}^{-m}}\right)
$$

where $\mathcal{M}=\mu_{\mathfrak{G}}^{-1}(0)$ is defined as in (4.3.1) with open subvariety

$$
\mathcal{M}^{\text {cyc }}=\{(X, Y, v, w) \in \mathcal{M}: \mathbb{C}[X, Y] v=V\} .
$$

In more detail, $\mathcal{M}^{\text {cyc }}$ is a smooth $G L(V)$-variety and the map $j: \mathcal{M}^{\text {cyc }} \hookrightarrow \mathcal{M}$ is a $G L(V)$-equivariant Zariski open imbedding. The map $p$ in (7.2.1) is a universal geometric quotient morphism that makes $\mathcal{M}^{\text {cyc }}$ a principal $G L(V)$-bundle over $\mathrm{Hilb}^{n} \mathbb{C}^{2}$ (see $\mathrm{Na}$, Proof of Theorem 1.9]). Finally, the penultimate isomorphism in (7.2.1) was proved in [Ha2, Proposition 2.6] while the last isomorphism follows from (4.3.2).

7.3. We can also construct Hilb $^{n} \mathbb{C}^{2}$ via

$$
T^{*} \mathfrak{X}=\left\{(X, Y, v, w) \in \mathfrak{g} \times \mathfrak{g} \times V^{\circ} \times V^{*}: w(v)=0\right\} / \mathbb{C}^{\times},
$$

where the action of $\mathbb{C}^{\times}$arises from the scalar matrices in $G L(V)$; thus it acts only on $V^{\circ} \times V^{*}$. In more detail, since $\operatorname{Tr}([X, Y]+v w)=w(v)$, then, as in (4.3),

$$
\mu_{\mathfrak{X}}^{-1}(0) \stackrel{\text { def }}{=}\left\{(X, Y, v, w) \in \mathfrak{g} \times \mathfrak{g} \times V_{27}^{\circ} \times V^{*}:[X, Y]+v w=0\right\} / \mathbb{C}^{\times}
$$


is a closed subvariety of $T^{*} \mathfrak{X}$. Inside $\mu_{\mathfrak{X}}^{-1}(0)$ we have the the Zariski open subset

$$
\mu_{\mathfrak{X}}^{-1}(0)^{\text {cyc }} \stackrel{\text { def }}{=}\left\{(X, Y, v, w) \in \mu_{\mathfrak{X}}^{-1}(0): v \in V^{\circ} \text { and } \mathbb{C}[X, Y] v=V\right\} \subset \mu_{\mathfrak{X}}^{-1}(0) .
$$

As before, we have a diagram schemes

$$
\mu_{\mathfrak{X}}^{-1}(0) \stackrel{j_{\mathfrak{X}}}{\longrightarrow} \mu_{\mathfrak{X}}^{-1}(0)^{\text {cyc }} \stackrel{p_{\mathfrak{X}}}{\longrightarrow} \operatorname{Hilb}^{n} \mathbb{C}^{2}
$$

where $p_{\mathfrak{X}}$ is a universal geometric quotient making a principal $P G L(V)$-bundle. There is, moreover, a commutative diagram

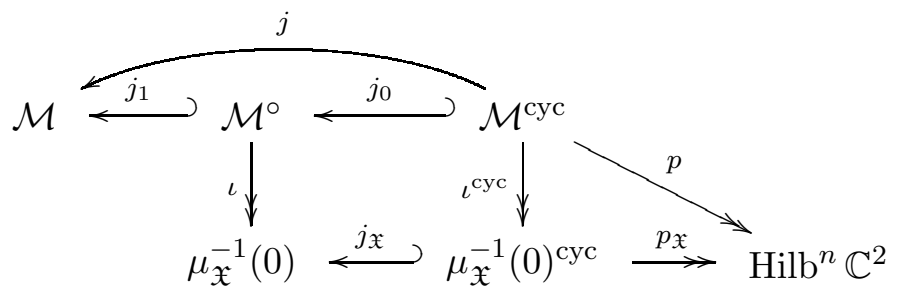

where $\mathcal{M}^{\circ}=\{(X, Y, v, w) \in \mathcal{M}: v \neq 0\}$ and the vertical maps $\iota, \iota^{\text {cyc }}$ are principal $\mathbb{C}^{\times}$-bundles.

7.4. We will require a special case of the following well-known proposition, although since we could not find a proof in the literature we include one here.

Let $X$ be an affine variety with a rational action of a reductive group $G$. Fix a character $\chi: G \longrightarrow \mathbb{C}^{\times}$and define the Zariski open set of semistable points to be, cf. e.g. [Mu], ch. 6-7,

$$
X^{s s}=\left\{x \in X: \text { there exists } m>0 \text { and } f \in \mathbb{C}[X]^{\chi^{m}} \text { such that } f(x) \neq 0\right\},
$$

and let $j: X^{s s} \hookrightarrow X$ be the inclusion. By definition the G.I.T. quotient $X / /{ }_{\chi} G$ is Proj $B$ where $B$ is the graded algebra $B=\bigoplus_{m \geq 0} \mathbb{C}[X]^{\chi^{m}}$. Write $\operatorname{Coh}^{G}(Y)$ for the abelian category of $G$-equivariant coherent sheaves on a $G$-variety $Y$.

Proposition. (1) For any $\mathscr{S} \in \mathrm{Coh}^{G}(X)$, there exists an integer $m(\mathscr{S})$ such that the restriction map induces an isomorphism

$$
j^{*}: \Gamma(X, \mathscr{S})^{\chi^{m}} \stackrel{\sim}{\longrightarrow} \Gamma\left(X^{s s}, j^{*} \mathscr{S}\right)^{\chi^{m}} \quad \text { for all } m \geq m(\mathscr{S}) .
$$

(2) If the orbit map $p: X^{s s} \longrightarrow X / /{ }_{\chi} G$ is a principal $G$-bundle then there is a natural isomorphism

$$
j^{*} \mathscr{S} \cong p^{*} \circ \mathbb{F}\left(\oplus_{m \geq 0} \Gamma(X, \mathscr{S})^{\chi^{m}}\right)
$$

of functors from $\mathrm{Coh}^{G}(X)$ to $\mathrm{Coh}^{G}\left(X^{s s}\right)$.

Proof. (1) Let $Z=X \backslash X^{s s}$, a closed subvariety of $X$. There is an exact sequence

$$
\Gamma_{Z}(X, \mathscr{S}) \longrightarrow \Gamma(X, \mathscr{S}) \longrightarrow \Gamma\left(X^{s s}, j^{*} \mathscr{S}\right) \longrightarrow H_{Z}^{1}(X, \mathscr{S}) .
$$

All modules in this sequence are rational $G$-modules, so taking $\chi^{m}$ semi-invariants produces another exact sequence. We claim that both $\Gamma_{Z}(X, \mathscr{S})^{\chi^{m}}$ and $H_{Z}^{1}(X, \mathscr{S})^{\chi^{m}}$ are 
zero for $m \gg 0$. Once we have shown this, then it follows that the map $\Gamma(X, \mathscr{S})^{\chi^{m}} \rightarrow$ $\Gamma\left(X^{s s}, j^{*} \mathscr{S}\right)^{\chi^{m}}$ is an isomorphism for large enough $m$, thus confirming (1).

To prove the claim, we define $S=\mathbb{C}[X \times \mathbb{C}]^{G}$ where $G$ acts on $X \times \mathbb{C}$ by $g \cdot(x, \lambda)=$ $\left(g \cdot x, \chi^{-1}(g) \lambda\right)$. It follows that $S$ is a finitely generated and so we can find a finite set of homogeneous elements $f_{1}, \ldots, f_{n}$ in $S_{+}$that generate the algebra $S$ over $S_{0}=\mathbb{C}[X]^{G}$. Let $I$ be the ideal of $\mathbb{C}[X]$ generated by $f_{1}, \ldots, f_{n}$ and observe that $S$ has been constructed so that

$$
Z=\left\{x \in X: f(x)=0 \text { for all } f \in S_{+}\right\}=\left\{x \in X: f_{i}(x)=0 \text { for } 1 \leq i \leq n\right\} .
$$

So to calculate the local cohomology groups $H_{Z}^{i}$ it is enough to calculate the $H_{I}^{i}$.

Let $R=\mathbb{C}[X], B=\bigoplus_{m \geq 0} \mathbb{C}[X]^{\chi^{m}}$ and $M=\Gamma(\mathcal{M}, \mathscr{S})$. We need to calculate the homology of the complex

$$
\begin{aligned}
0 \longrightarrow M \longrightarrow\left(\bigoplus_{i} R_{f_{i}}\right) \otimes_{R} M \longrightarrow\left(\bigoplus_{i<j} R_{f_{i} f_{j}}\right) \otimes_{R} M & \longrightarrow \cdots \\
\cdots & \longrightarrow R_{f_{1} \cdots f_{r}} \otimes_{R} M \longrightarrow 0 .
\end{aligned}
$$

As each $f_{i} \in B$, we can replace $R$ by $B$ in the above sequence. Since we are going to take $\chi$ semi-invariants it is enough for us to study the complex with $M$ replaced by $N=\bigoplus_{m \in \mathbb{Z}} M^{\chi^{m}}$. Set $N^{+}=\bigoplus_{m \geq 0} M^{\chi^{m}}$. Since $N / N^{+}$is $f_{i_{1}} \ldots f_{i_{r}}$-torsion for $r \geq 1$, tensoring the short exact sequence $0 \longrightarrow N_{+} \longrightarrow N \longrightarrow N / N_{+} \longrightarrow 0$ by $S_{f_{i_{1}} \ldots f_{i_{r}}}$ shows that $S_{f_{i_{1}} \ldots f_{i_{r}}} \otimes_{S} N_{+} \cong S_{f_{i_{1}} \ldots f_{i_{r}}} \otimes_{S} N$. Thus for our calculation we can even replace $N$ by $N_{+}$and we need only calculate the local cohomology groups $H_{S_{+}}^{i}\left(N_{+}\right)^{\chi^{m}}$. Now $N_{+}$is a finitely generated graded $S$-module so thanks to [BS, 15.1.5], these groups vanish for $m \gg 0$.

(2) This follows from (1) and the projection formula. In more detail, by part (1) we have

$$
\mathbb{F}\left(\bigoplus_{m \geq 0} \Gamma(X, \mathscr{S})^{\chi^{m}}\right)=\mathbb{F}\left(\bigoplus_{m \geq 0} \Gamma\left(X^{s s}, j^{*} \mathscr{S}\right)^{\chi^{m}}\right)
$$

Since $p$ defines a principal $G$-bundle we deduce that $p^{*}\left[\left(p_{*} j^{*} \mathscr{S}\right)^{G}\right] \cong j^{*} \mathscr{S}$ (see [SGA1, Chapter VII, Section 1], again). Thus it is sufficient to check that

$$
\left(p_{*} \mathcal{F}\right)^{G} \cong \mathbb{F}\left(\bigoplus_{m \geq 0} \Gamma\left(X^{s s}, \mathcal{F}\right)^{\chi^{m}}\right) \quad \text { for any } \mathcal{F} \in \operatorname{Coh}^{G}\left(X^{s s}\right) .
$$

But by definition $\left(p_{*} \mathcal{F}\right)^{G} \cong \mathbb{F}\left(\bigoplus_{m \geq 0} \Gamma\left(X / / \chi G,\left(p_{*} \mathcal{F}\right)^{G}(m)\right)\right.$. Now

$$
\begin{aligned}
\left(p_{*} \mathcal{F}\right)^{G}(m) \cong\left(p_{*} \mathcal{F}(m)\right)^{G} \cong & p_{*}\left(\mathcal{F} \otimes \mathcal{O}_{X^{s s}} p^{*} \mathcal{O}_{X / / \chi}(m)\right)^{G} \\
& \cong p_{*}\left(\mathcal{F} \otimes \mathbb{C}_{\chi^{-m}}\right)^{G} \cong\left(p_{*} \mathcal{F}\right)^{\chi^{m}}
\end{aligned}
$$

The proof is now completed by the following isomorphisms

$$
\Gamma\left(X / / \chi G,\left(p_{*} \mathcal{F}\right)^{G}(m)\right) \cong \Gamma\left(X / / \chi G,\left(p_{*} \mathcal{F}\right)^{\chi^{m}}\right) \cong \Gamma\left(X / / \chi G, p_{*} \mathcal{F}\right)^{\chi^{m}} \cong \Gamma\left(X^{s s}, \mathcal{F}\right)^{\chi^{m}} .
$$


7.5. Characteristic cycles. We assume that $c+m$ is good for all $m \in \mathbb{Z}_{\geq 0}$. Given a finitely generated left $\mathrm{U}_{c}$-module $M$, there are two ways to associate to $M$ an algebraic cycle in Hilb $^{n} \mathbb{C}^{2}$.

GS construction (see [GS2, Section 2.7] for more details). Take a good filtration on $M$ and let $\Lambda$ be the induced tensor product filtration on each $\mathfrak{M}_{m}=\left({ }_{c+m} \mathrm{P}_{c}\right) \otimes \mathbf{U}_{c} M$ and hence on $\mathfrak{M}=\bigoplus_{m \geq 0} \mathfrak{M}_{m}$. By Theorem 1.7 $\operatorname{gr}\left({ }_{c+\ell+m} \mathrm{P}_{c+m}\right)=A^{\ell}$ for all $\ell, m \geq 0$ and it follows easily that $\operatorname{gr}_{\Lambda} \mathfrak{M}=\bigoplus \operatorname{gr}_{\Lambda} \mathfrak{M}_{m}$ is a graded module over $A=\bigoplus A^{\ell}$. Let $\mathbb{F}(\operatorname{gr} \mathfrak{M})$ denote the corresponding coherent sheaf on Proj $A=\operatorname{Hilb}^{n} \mathbb{C}^{2}$. We define $\operatorname{ch}^{G S}(M)$ to be the characteristic cycle of $\mathbb{F}(\operatorname{gr} \mathfrak{M})$. In other words $\mathrm{ch}^{G S}(M)$ is the characteristic variety of $\mathbb{F}(\operatorname{gr} \mathfrak{M})$, counting multiplicities, see [GS2, (2.7.1)].

GG construction (see [GG, Section 7.5] for the details). Recall the definition of ${ }^{\top} \widetilde{\mathbb{H}}_{c}$ from (6.6) and consider the left $\mathscr{D}(\mathfrak{G})$-module

$$
{ }^{\top} \widetilde{\mathbb{H}}_{c}(M)=\left(\mathscr{D}_{-c}(\mathfrak{X}) / \mathscr{D}_{-c}(\mathfrak{X}) \tau\left(I_{-c}\right)\right) \otimes_{U_{-c-1}} \Omega_{c}(M) .
$$

Let $C C\left({ }^{\top} \widetilde{\mathbb{H}}_{c}(M)\right) \subset T^{*} \mathfrak{X}$ be the characteristic cycle of that $\mathscr{D}_{-c}(\mathfrak{X})$-module, a closed $P G L(V)$-invariant algebraic cycle set-theoretically contained in $\mu_{\mathfrak{X}}^{-1}(0)$. Following $\mathrm{GG}$, Section 7.5]2 and in the notation of (7.2), define $\mathrm{ch}^{G G}(M)$ to be the unique algebraic cycle in $\operatorname{Hilb}^{n} \mathbb{C}^{2}$ such that one has $j_{\mathfrak{X}}^{*} C C\left({ }^{\top} \widetilde{\mathbb{H}}_{c}(M)\right)=p_{\mathfrak{X}}^{*}$ ch $^{G G}(M)$ inside $\mathcal{M}^{\text {cyc }}$.

7.6. We are now ready to prove the following slight strengthening of Theorem 1.10 from the introduction.

Theorem. Assume that $c \in \mathbb{C} \backslash \mathbb{Z}_{<0}$ is chosen such that $c+m$ is good for all integers $m \geq 0$. If $n=2$ assume that $n c \notin \mathbb{Z}$. Then, for any finitely generated $\cup_{c}$-module $M$, one has an equality of algebraic cycles $\mathrm{ch}^{G S}(M)=\mathrm{ch}^{G G}(M)$.

Proof. Our hypotheses ensure that $n c \notin \mathbb{Z}_{<0}$, so Corollary 6.6 is available to us.

Put $F=\mathfrak{D}_{-c} \otimes \mathrm{U}_{-c-1} \Omega_{c}(M)$ and note that $F$ is $G L(V)$-equivariant since the same is true of $\mathfrak{D}_{-c}$. For any integer $m \geq 0$ the $\mathscr{D}(\mathfrak{G})$-module structure on $F$ induces a natural $\mathscr{D}_{-c-m}(\mathfrak{X})$-module structure on $F^{(-m)}$. We compute

$$
\begin{aligned}
F^{(-m)} & =\left[\mathfrak{D}_{-c} \otimes_{\mathbf{U}_{-c-1}} \Omega_{c}(M)\right]^{(-m)}={ }_{-c-m} \mathfrak{D}_{-c} \otimes_{\mathbf{U}_{-c-1}} \Omega_{c}(M) \\
& ={ }_{-c-m} \mathfrak{D}_{-c} \otimes_{\mathscr{D}_{-c}(\mathfrak{X})}\left(\frac{\mathscr{D}_{-c}(\mathfrak{X})}{\mathscr{D}_{-c}(\mathfrak{X}) \tau\left(I_{-c}\right)}\right) \otimes_{\mathbf{U}_{-c-1}} \Omega_{c}(M)=\mathbb{S}^{m}{ }^{\top} \widetilde{\mathbb{H}}_{c}(M),
\end{aligned}
$$

where in the last equality we have used the analogue of Lemma $6.7(1)$ for the $\mathscr{D}_{-c}(\mathfrak{X})$ module ${ }^{\top} \widetilde{\mathbb{H}}_{c}(M)$. Hence, taking $S L$-invariants and using Corollary 6.6, we deduce that

$$
\begin{aligned}
\Omega_{-c-m-1}\left(\left[F^{(-m)}\right]^{S L}\right) & =\Omega_{-c-m-1}\left(\left[\mathbb{S}^{m} \circ \widetilde{\mathbb{H}}_{c}(M)\right]^{S L}\right) \\
& =\widetilde{\mathbb{H}}_{c-m} \circ \mathbb{S}^{m}{ }^{\top} \widetilde{\mathbb{H}}_{c}(M)={ }_{c+m} \mathrm{P}_{c} \otimes \mathbf{U}_{c} M .
\end{aligned}
$$

\footnotetext{
${ }^{2}$ Actually, we have twisted by $\Omega_{c}$
} 
Pick a good filtration on $M$ and let $\Lambda$ denote the induced tensor product filtration on ${ }_{c+m} \mathrm{P}_{c} \otimes \mathbf{U}_{c} M$. Writing 'Supp(-)' for the support-cycle, we have

$$
\operatorname{ch}^{G S}(M)=\operatorname{Supp} \mathbb{F}\left(\operatorname{gr}_{\Lambda} \mathfrak{M}\right) \quad \text { where } \quad \mathfrak{M}=\oplus_{m \geq 0}\left({ }_{c+m} \mathrm{P}_{c} \otimes \mathrm{U}_{c} M\right) .
$$

Since $\phi_{c}$ preserves the differential operator filtration, the filtration on $M$ also gives a filtration on $\Omega_{c}(M)$, and hence a tensor product filtration $\nu$ on $F$. Observe that, viewed as a $\mathscr{D}(\mathfrak{G})$-module, this is a good filtration on $F$ since, by [GS1, Lemma 6.7(2)], $\operatorname{gr}_{\nu} F$ is a homomorphic image of $\operatorname{gr}_{\Gamma} \mathfrak{D}_{-c} \otimes_{\operatorname{gr} U_{c-1}} \operatorname{gr}\left(\Omega_{c}(M)\right)$. The filtration on $F$ is $G L(V)$-stable and, for any $m \geq 0$, it restricts to a filtration on each of the subspaces $\left[F^{(-m)}\right]^{S L} \subset F^{(-m)} \subset F$. Now, after applying $\Omega_{-c-m-1}$, the composite isomorphism in (7.6.1) transports this filtration on $\Omega_{-c-m-1}\left(\left[F^{(-m)}\right]^{S L}\right)$ to a certain $\nu$-filtration on ${ }_{c+m} \mathrm{P}_{c} \otimes \mathrm{U}_{c} M$. However, this need not equal to the $\Lambda$-filtration introduced earlier.

Since the action of $G L(V)$ on $F$ is locally finite, taking the associated graded $\operatorname{gr}_{\nu}(-)$ commutes with taking $G L(V)$-semi-invariants. Hence we have

$$
\begin{aligned}
\operatorname{gr}_{\nu} \mathfrak{M} & =\bigoplus_{m \geq 0} \operatorname{gr}_{\nu}\left({ }_{c+m} \mathrm{P}_{c} \otimes \mathrm{U}_{c} M\right)=\bigoplus_{m \geq 0} \operatorname{gr}_{\nu}\left(\Omega_{-c-m-1}\left(\left[F^{(-m)}\right]^{S L}\right)\right) \\
& =\bigoplus_{m \geq 0} \operatorname{gr} \Omega_{-c-m-1}\left(\operatorname{gr}_{\nu}\left(\left[F^{(-m)}\right]^{S L}\right)\right)=\bigoplus_{m \geq 0}\left[\left(\operatorname{gr}_{\nu} F\right)^{(-m)}\right]^{S L}
\end{aligned}
$$

where for the last equality we used that gr $\Omega_{-c-m-1}$ is the identity map since the associated graded of the mapping $z \mapsto \phi_{-c-m-1}\left(\delta^{-1} z \delta\right)$ is the identity on $\mathbb{C}\left[\mathfrak{h} \times \mathfrak{h}^{*}\right]^{W} e$. Since $\operatorname{gr}_{\nu} F$ is a finitely generated $\operatorname{gr}_{\Gamma} \mathscr{D}(\mathfrak{G})=\mathbb{C}\left[T^{*} \mathfrak{G}\right]$-module the final object of (7.6.3) is a finitely generated graded $A$-module. It follows that the $\nu$-filtration on $\mathfrak{M}$ is good and so the associated graded modules $\operatorname{gr}_{\Lambda} \mathfrak{M}$ and $\operatorname{gr}_{\nu} \mathfrak{M}$ give rise to the same class in the Grothendieck semigroup of the category $A$-grmod. We conclude that

$$
\text { Supp } \mathbb{F}\left(\operatorname{gr}_{\Lambda} \mathfrak{M}\right)=\operatorname{Supp} \mathbb{F}\left(\operatorname{gr}_{\nu} \mathfrak{M}\right)=\operatorname{Supp} \mathbb{F}\left(\bigoplus_{m \geq 0}\left[\operatorname{gr}_{\nu} F^{(-m)}\right]^{S L}\right) \text {. }
$$

Since $T^{*} \mathfrak{G}$ is affine, we can write $\operatorname{gr}_{\nu} F=\Gamma\left(T^{*} \mathfrak{G}, \mathcal{F}\right)$ for a unique coherent sheaf $\mathcal{F}$ supported on the subvariety $\mathcal{M} \subset T^{*} \mathfrak{G}$. The sheaf $\mathcal{F}$ is automatically $G L(V)$-equivariant since $F$ is. Taking $S L$-invariants and applying (7.4.1), we deduce that, for large enough $m$,

$$
\left[\operatorname{gr}_{\nu} F^{(-m)}\right]^{S L}=\left[\Gamma\left(T^{*} \mathfrak{G}, \mathcal{F}\right)^{(-m)}\right]^{S L}=\Gamma(\mathcal{M}, \mathcal{F})^{\operatorname{det}^{-m}}=\Gamma\left(\mathcal{M}^{\text {cyc }}, \mathcal{F}\right)^{\operatorname{det}^{-m}}
$$

Thus, in the category $\operatorname{Coh}\left(\operatorname{Hilb}^{n} \mathbb{C}^{2}\right)$, we have the isomorphisms

$$
\mathbb{F}\left(\operatorname{gr}_{\nu} \mathfrak{M}\right) \cong \mathbb{F}\left(\bigoplus_{m \geq 0}\left[\operatorname{gr}_{\nu} F^{(-m)}\right]^{S L}\right) \cong \mathbb{F}\left(\bigoplus_{m \geq 0} \Gamma\left(\mathcal{M}^{\mathrm{cyc}}, \mathcal{F}\right)^{\operatorname{det}^{-m}}\right) .
$$

Applying the isomorphism of functors in (7.4.2), we obtain

$$
p^{*} \mathbb{F}\left(\operatorname{gr}_{\nu} \mathfrak{M}\right) \cong p^{*} \mathbb{F}\left(\bigoplus_{m \geq 0} \Gamma\left(\mathcal{M}^{\mathrm{cyc}}, \mathcal{F}\right)^{\operatorname{det}^{-m}}\right)=j^{*} \mathcal{F}
$$


Using this and (7.6.2) and (7.6.3) , we finally obtain a chain of equalities of algebraic cycles

$$
p^{*}\left(\operatorname{ch}^{G S}(M)\right)=\operatorname{Supp}\left(p^{*} \mathbb{F}\left(\operatorname{gr}_{\nu} \mathfrak{M}\right)\right)=\operatorname{Supp}\left(j^{*} \mathcal{F}\right) .
$$

It remains to prove that $\operatorname{Supp}\left(j^{*} \mathcal{F}\right)=p^{*}\left(\mathrm{ch}^{G G}(M)\right)$. This is now completely formal. By the commutative diagram (7.3.1) and the definition of $\mathrm{ch}^{G G}(M)$ we have

$$
p^{*}\left(\operatorname{ch}^{G G}(M)\right)=\left(\iota^{\mathrm{cyc}}\right)^{*} p_{\mathfrak{X}}^{*}\left(\operatorname{ch}^{G G}(M)\right)=\left(\iota^{\mathrm{cyc}}\right)^{*} j_{\mathfrak{X}}^{*} C C\left({ }^{\top} \widetilde{\mathbb{H}}_{c}(M)\right)=j_{0}^{*} \iota^{*} C C\left({ }^{\top} \widetilde{\mathbb{H}}_{c}(M)\right) .
$$

Since $\operatorname{Supp}\left(j^{*} \mathcal{F}\right)=j^{*} \operatorname{Supp}(\mathcal{F})=j_{0}^{*} \operatorname{Supp}\left(j_{1}^{*} \mathcal{F}\right)$ it is $\operatorname{sufficient}$ to $\operatorname{show}$ that $\operatorname{Supp}\left(j_{1}^{*} \mathcal{F}\right)=$ $\iota^{*} C C\left({ }^{\top} \widetilde{\mathbb{H}}_{c}(M)\right)$. We compute

$$
\begin{aligned}
\iota^{*} C C\left({ }^{\top} \widetilde{\mathbb{H}}_{c}(M)\right)=\iota^{*} \operatorname{Supp}\left(\left(\operatorname{gr}_{\nu} F\right)^{\mathbb{C}^{\times}}\right) & =\operatorname{Supp}\left(\iota^{*}\left(\operatorname{gr}_{\nu} F\right)^{\mathbb{C}^{\times}}\right) \\
& =\operatorname{Supp}\left(j_{1}^{*} \operatorname{gr}_{\nu} F\right)=\operatorname{Supp}\left(j_{1}^{*} \mathcal{F}\right) .
\end{aligned}
$$

\section{Appendix: The RADial Parts MaP}

8.1. As was mentioned earlier, in this appendix we will give the details behind Theorem 2.8, since it does not exactly follow from the results in [GG]. Thus, in the notation of Section 4.2 our aim is to prove:

Theorem. The radial components map $\mathfrak{R}=\mathfrak{R}_{c}: \mathscr{D}\left(\mathfrak{G}^{\mathrm{cyc}}\right)^{G} \rightarrow \mathscr{D}(\mathfrak{h} / W)$ given by

$$
\mathfrak{R}(D)=\mathbf{s}^{c} \circ\left(\left.D\right|_{\mathcal{O}\left(\mathfrak{G}^{\mathrm{cyc}}, c\right)}\right) \circ \mathbf{s}^{-c} \quad \text { for } D \in \mathscr{D}(\mathfrak{G})^{G}
$$

induces a filtered isomorphism $\overline{\mathfrak{R}}: \mathfrak{D}_{c}^{G}=\left(\mathscr{D}(\mathfrak{G}) / \mathscr{D}(\mathfrak{G}) \cdot I_{c}\right)^{G} \stackrel{\sim}{\longrightarrow} \mathrm{U}_{c-1}$.

Consequently, the associated graded map gr $\overline{\mathfrak{R}}: \operatorname{gr} \mathfrak{D}_{c}^{G} \rightarrow \operatorname{gr} \mathrm{U}_{c-1}=\mathbb{C}\left[\mathfrak{h} \times \mathfrak{h}^{*}\right]^{W}$ is also an isomorphism.

The proof of this result closely follows the proof of the analogous results in EG and [BFG] (see, in particular [BFG, Proposition 5.4.1]), so the important point here is to determine which spherical algebra $\bigcup_{d}$ contains $\operatorname{Im}(\mathfrak{R})$.

To begin, let $\Delta_{\mathfrak{g}}$ be the second order Laplacian on $\mathfrak{g}$ associated to non-degenerate invariant bilinear form $(-,-)$ and identify $\Delta_{\mathfrak{g}}$ with $\Delta_{\mathfrak{g}} \otimes 1 \in \mathscr{D}(\mathfrak{G})$ acting trivially in the $V$-direction. Write $\Delta_{\mathfrak{h}}$ for the analogous Laplacian on $\mathfrak{h}$. As usual, we let $\left\{e_{\alpha}: \alpha \in R\right\}$ denote the root vectors for $\mathfrak{g}$, normalised so that $\left(e_{\alpha}, e_{-\alpha}\right)=1$ and set $h_{\alpha}=\left[e_{\alpha}, e_{-\alpha}\right]$ for such $\alpha$. Now $\Delta_{\mathfrak{g}} \in \mathscr{D}(\mathfrak{g})^{G}$ and, as in the analogous computations in [EG, Proposition 6.2], the key to proving the theorem is to compute $\mathfrak{R}\left(\Delta_{\mathfrak{g}}\right)$.

To do this we slightly change our perspective on $\mathfrak{R}$. Fix a scalar $w \in \mathbb{C}$ (which will eventually become $w=-c)$ and let $\mathfrak{G}^{\text {reg }}=\left\{(X, v) \in \mathfrak{G}^{\text {cyc }}: X \in \mathfrak{g}^{\text {rs }}\right\}$, where $\mathfrak{g}^{\text {rs }} \supset \mathfrak{h}^{\text {reg }}$ denotes the regular semisimple elements. As in Section 4.2 or [BFG, Section 5.4] the projection $\mathfrak{G} \rightarrow \mathfrak{g}$ induces an isomorphism $\rho^{*}: \mathbb{C}\left[\mathfrak{h}^{\text {reg }} / W\right] \rightarrow \mathbb{C}\left[\mathfrak{G}^{\text {reg }}\right]^{G}$. For $D \in \mathscr{D}(\mathfrak{G})^{G}$ we define $\operatorname{Rad}(D) \in \mathscr{D}\left(\mathfrak{h}^{\text {reg }}\right)^{W}=\mathscr{D}\left(\mathfrak{h}^{\text {reg }} / W\right)$ to be $\mathfrak{R}_{-w}(D)$; equivalently,

$$
\operatorname{Rad}(D)(f)=\left.\mathbf{s}^{-w}\left(D\left(\mathbf{s}^{w}\left(\rho^{*} f\right)\right)\right)\right|_{\mathfrak{h}^{\text {reg }}} \quad \text { for } f \in \mathbb{C}\left[\mathfrak{h}^{\text {reg }} / W\right]
$$


8.2. The radial part of the Laplacian. We wish to compute $\operatorname{Rad}\left(\Delta_{\mathfrak{g}}\right)$ and we begin by following the proof of [EG, Proposition 6.2]. As observed there, we have the expansion

$$
\Delta_{\mathfrak{g}}=\Delta_{\mathfrak{h}}+\sum_{\alpha \in R} \frac{\partial^{2}}{\partial e_{\alpha} \partial e_{-\alpha}}
$$

and we begin by understanding the final term of this equation.

The differential of the $G$-action on $\mathfrak{G}^{\text {reg }}$ gives an action of $\mathfrak{g}$ and $U(\mathfrak{g})$ on $\mathbb{C}\left[\mathfrak{G}^{\text {reg }}\right]$ which we will write as $x \cdot f$ for $x \in U(\mathfrak{g})$ and $f \in \mathbb{C}\left[\mathfrak{G}^{\text {reg }}\right]$. By definition, $\rho^{*} f \in \mathbb{C}\left[\mathfrak{G}^{\text {reg }}\right]^{G}$ for $f \in \mathbb{C}\left[\mathfrak{h}^{\text {reg }} / W\right]$ and so $g \stackrel{\text { def }}{=} \mathbf{s}^{w} \rho^{*}(f)$ is a $\left(\operatorname{det}^{-w}\right)$-semi-invariant function, since the same is true of $\mathbf{s}^{w}$. Therefore, for $X \in \mathfrak{h}^{\text {reg }}, u e_{\alpha} \in \mathbb{C} e_{\alpha}$ and $v \in V$ we have

$$
\begin{aligned}
\left(e^{t e_{\alpha}} \cdot g\right)\left(X+u e_{-\alpha}, v\right)= & \left(\operatorname{det}\left(e^{t e_{\alpha}}\right)\right)^{-w} g\left(X+u e_{-\alpha}, v\right) \\
& =\left(1-w t \operatorname{Tr}\left(e_{\alpha}\right)+O\left(t^{2}\right)\right) g\left(X+u e_{-\alpha}, v\right) . .
\end{aligned}
$$

On the other hand,

$$
\begin{gathered}
\left(e^{t e_{\alpha}} \cdot g\right)\left(X+u e_{-\alpha}, v\right)=g\left(\left(\operatorname{Ad} e^{-t e_{\alpha}}\right)\left(X+u e_{-\alpha}\right), e^{-t e_{\alpha}} \cdot v\right) \\
=g\left(X+u e_{-\alpha}+t\left[e_{\alpha}, X\right]-t u\left[e_{\alpha}, e_{-\alpha}\right]+O\left(t^{2}\right), v-t e_{\alpha} \cdot v+O\left(t^{2}\right)\right) \\
=g\left(X+u e_{-\alpha}-t \alpha(X) e_{\alpha}-t u h_{\alpha}+O\left(t^{2}\right), v-t e_{\alpha} \cdot v+O\left(t^{2}\right)\right) .
\end{gathered}
$$

Now clearly $0=\operatorname{Tr}\left(e_{\alpha}\right)$ and so after equating the last two equations, applying $d / d t$, and then setting $t=0$, we obtain

$$
0=\alpha(X) \frac{\partial}{\partial e_{\alpha}} g\left(X+u e_{-\alpha}, v\right)-u \frac{\partial}{\partial h_{\alpha}} g\left(X+u e_{-\alpha}, v\right)-e_{\alpha} \cdot g\left(X+u e_{-\alpha}, v\right) .
$$

Rewriting this gives

$$
\frac{\partial}{\partial e_{\alpha}} g\left(X+u e_{-\alpha}, v\right)=\frac{1}{\alpha(X)}\left(u \frac{\partial}{\partial h_{\alpha}} g\left(X+u e_{-\alpha}, v\right)+e_{\alpha} \cdot g\left(X+u e_{-\alpha}, v\right)\right) .
$$

Now apply $d / d u$ to this equation and then set $u=0$ to give

$$
\frac{\partial^{2}}{\partial e_{-\alpha} \partial e_{\alpha}} g(X, v)=\frac{1}{\alpha(X)}\left[\frac{\partial}{\partial h_{\alpha}} g(X, v)+\frac{\partial}{\partial e_{-\alpha}}\left(e_{\alpha} \cdot g(X, v)\right)\right] .
$$

Applying (8.2.2) with $e_{\alpha}$ replaced by $e_{-\alpha}$ gives

$$
\frac{\partial}{\partial e_{-\alpha}} g\left(X, v+t e_{\alpha} \cdot v\right)=-\frac{1}{\alpha(X)} e_{-\alpha} \cdot g\left(X, v+t e_{\alpha} \cdot v\right) .
$$

Therefore $\frac{\partial}{\partial e_{-\alpha}}\left(e_{\alpha} \cdot g(X, v)\right)=-\frac{1}{\alpha(X)} e_{-\alpha} e_{\alpha} \cdot g(X, v)$ and so (8.2.3) becomes

$$
\frac{\partial^{2}}{\partial e_{-\alpha} \partial e_{\alpha}} g(X, v)=\frac{1}{\alpha(X)} \frac{\partial}{\partial h_{\alpha}} g(X, v)-\frac{1}{\alpha(X)^{2}} e_{-\alpha} e_{\alpha} \cdot g(X, v)
$$

for $X \in \mathfrak{h}^{\text {reg }}$ and $v \in V$. 
Next we calculate $e_{-\alpha} e_{\alpha} \cdot g(X, v)$ for $X \in \mathfrak{h}^{\text {reg. }}$. Recall that $g=\mathbf{s}^{w} \rho^{*}(f)$ with $\rho^{*} f \in \mathbb{C}\left[\mathfrak{G}^{\text {reg }}\right]^{G}$. As noted in $[\mathrm{BFG}$, Section 5.4], projection onto the first term gives an isomorphism $\mathbb{C}[\mathfrak{G}]^{G} \stackrel{\sim}{\longrightarrow} \mathbb{C}[\mathfrak{g}]^{G}$ and so $\left.e_{-\alpha} \cdot \rho^{*}(f)\right|_{\mathfrak{h}^{\text {reg }}}=\left.e_{\alpha} \cdot \rho^{*}(f)\right|_{\mathfrak{h}^{\text {reg }}}=0$. Thus

$$
\left.e_{-\alpha} e_{\alpha} \cdot \mathbf{s}^{w} \rho^{*}(f)\right|_{\mathfrak{h}^{\text {reg }}}=\left(\left.e_{-\alpha} e_{\alpha} \cdot \mathbf{s}^{w}\right|_{\mathfrak{h}^{\text {reg }}}\right) f \text {. }
$$

Now for $X=\left(x_{1}, \ldots, x_{n}\right) \in \mathfrak{h}^{\text {reg }}$ and $v=\left(v_{1}, \ldots, v_{n}\right) \in V$ we have

$$
\mathbf{s}^{w}(X, v)=\prod_{i<j}\left(x_{i}-x_{j}\right)^{w}\left(v_{1} \cdots v_{n}\right)^{w} .
$$

If $\alpha$ is the elementary matrix $\alpha=E_{i j}$ then

$$
e_{\alpha} \cdot\left(v_{1}^{a_{1}} \cdots v_{n}^{a_{n}}\right)=a_{i} v_{1}^{a_{1}} \cdots v_{i}^{a_{i}-1} \cdots v_{j}^{a_{j}+1} \cdots v_{n}^{a_{n}} \quad \text { for any } a_{\ell} \in \mathbb{C} .
$$

Thus $\left.e_{-\alpha} e_{\alpha} \cdot \mathbf{s}^{w}\right|_{\mathfrak{h}^{\text {reg }}}=\left.w(w+1) \mathbf{s}^{w}\right|_{\mathfrak{h}^{\text {reg }}}$ and so

$$
\left.e_{-\alpha} e_{\alpha} \cdot g(X, v)\right|_{\mathfrak{h}^{\text {reg }}}=\left.w(w+1) g(X, v)\right|_{\mathfrak{h}^{\text {reg }}} \cdot
$$

Finally, observe that $\left.\mathbf{s}^{w}\right|_{\mathfrak{h}^{\text {reg }}}=\delta^{w}$. Thus combining (8.2.1), (8.2.4) and (8.2.6) produces the desired equation

$$
\operatorname{Rad}\left(\Delta_{\mathfrak{g}}\right)=\delta^{-w} \Delta_{\mathfrak{h}} \delta^{w}+\sum_{\alpha \in R} \frac{\delta^{-w}}{\alpha} \frac{\partial}{\partial h_{\alpha}} \delta^{w}-\sum_{\alpha \in R} \frac{w(w+1)}{\alpha^{2}} .
$$

8.3. Recall from (2.3) that we have identified $\mathrm{H}_{w}$ with its image in $\mathscr{D}\left(\mathfrak{h}^{\text {reg }}\right) * W$ under the Dunkl embedding. As in [EG, p. 281], we define a twisted Dunkl homomorphism $\Theta_{w}^{\text {spher }}: \mathbf{H}_{w} \rightarrow \mathscr{D}\left(\mathfrak{h}^{\text {reg }}\right) * W$ by $\Theta_{w}^{\text {spher }}(h)=\delta^{-w} h \delta^{w}$ for $h \in \mathbf{H}_{w}$.

Recall further the Calogero-Moser operator defined by the formula

$$
L_{w} \stackrel{\text { def }}{=} \Delta_{\mathfrak{h}}-\frac{1}{2} \sum_{\alpha \in R} w(w+1) \frac{(\alpha, \alpha)}{\alpha^{2}} .
$$

It was mentioned in [EG, pp. 281-2] that the Calogero-Moser operator lies in $\Theta_{w}^{\text {spher }}\left(\mathrm{H}_{w}\right)$. We take this opportunity to give a complete proof of this assertion. In this computation we write $\partial_{t}=\partial / \partial x_{t}$ for $t \geq 1$ and $x_{i j}=x_{i}-x_{j}$ for $i \neq j$. Then (2.3.1) gives

$$
\begin{aligned}
\sum_{t=1}^{n} D_{w}\left(y_{t}\right)^{2} e & =\sum_{t=1}^{n}\left(\left(\partial_{t}\right)^{2}-\sum_{j<i} w \frac{\left\langle y_{t}, x_{i j}\right\rangle}{x_{i j}}\left(\partial_{t}-s_{i j}\left(\partial_{t}\right)\right)\right) \\
& =\sum_{t=1}^{n}\left(\left(\partial_{t}\right)^{2}-2 \sum_{j<i} w \frac{\left\langle y_{t}, x_{i j}\right\rangle}{x_{i j}} \partial_{t}\right) .
\end{aligned}
$$

Now $\delta^{-w} \sum_{t=1}^{n} \partial_{t}=\sum_{t=1}^{n}\left(\partial_{t} \delta^{-w}+\sum_{j<i} w \frac{\left\langle y_{t}, x_{i j}\right\rangle}{x_{i j}} \delta^{-w}\right)$. Thus, we compute $\delta^{-w} \sum_{t=1}^{n}\left(\left(\partial_{t}\right)^{2}-2 \sum_{j<i} w \frac{\left\langle y_{t}, x_{i j}\right\rangle}{x_{i j}} \partial_{t}\right)$

$$
=\sum_{t=1}^{n}\left(\partial_{t} \delta^{-w} \partial_{t}+\sum_{j<i} w \frac{\left\langle y_{t}, x_{i j}\right\rangle}{x_{i j}} \delta^{-w} \partial_{t}-\delta^{-w} 2 \sum_{j<i} w \frac{\left\langle y_{t}, x_{i j}\right\rangle}{x_{i j}} \partial_{t}\right)
$$




$$
\begin{aligned}
& =\sum_{t=1}^{n}\left(\partial_{t} \delta^{-w} \partial_{t}-\sum_{j<i} w \frac{\left\langle y_{t}, x_{i j}\right\rangle}{x_{i j}} \delta^{-w} \partial_{t}\right) \\
& =\sum_{t=1}^{n}\left(\partial_{t}^{2} \delta^{-w}+\sum_{j<i} w \partial_{t} \frac{\left\langle y_{t}, x_{i j}\right\rangle}{x_{i j}} \delta^{-w}-\sum_{j<i} w \frac{\left\langle y_{t}, x_{i j}\right\rangle}{x_{i j}} \delta^{-w} \partial_{t}\right) \\
& =\sum_{t=1}^{n}\left(\partial_{t}^{2} \delta^{-w}+\sum_{j<i} w \frac{\left\langle y_{t}, x_{i j}\right\rangle}{x_{i j}} \partial_{t} \delta^{-w}-\sum_{j<i} w \frac{\left\langle y_{t}, x_{i j}\right\rangle^{2}}{x_{i j}^{2}} \delta^{-w}-\sum_{j<i} w \frac{\left\langle y_{t}, x_{i j}\right\rangle}{x_{i j}} \delta^{-w} \partial_{t}\right) \\
& =\sum_{t=1}^{n}\left(\partial_{t}^{2} \delta^{-w}+\sum_{j<i} w \frac{\left\langle y_{t}, x_{i j}\right\rangle}{x_{i j}}\left[\partial_{t}, \delta^{-w}\right]-\sum_{j<i} w \frac{\left\langle y_{t}, x_{i j}\right\rangle^{2}}{x_{i j}^{2}} \delta^{-w}\right) \\
& =\sum_{t=1}^{n}\left(\partial_{t}^{2} \delta^{-w}-\left(\sum_{j<i} w \frac{\left\langle y_{t}, x_{i j}\right\rangle}{x_{i j}}\right)^{2} \delta^{-w}-\sum_{j<i} w \frac{\left\langle y_{t}, x_{i j}\right\rangle^{2}}{x_{i j}^{2}} \delta^{-w}\right) \\
& =\sum_{t=1}^{n}\left(\partial_{t}^{2} \delta^{-w}-\sum_{\substack{i<j, k<\ell \\
(i, j) \neq(k, \ell)}} w^{2} \frac{\left\langle y_{t}, x_{i j}\right\rangle\left\langle y_{t}, x_{k \ell}\right\rangle}{x_{i j} x_{k \ell}} \delta^{-w}-\sum_{j<i}\left(w^{2}+w\right) \frac{\left\langle y_{t}, x_{i j}\right\rangle^{2}}{x_{i j}^{2}} \delta^{-w}\right) \\
& =\left(\sum_{t=1}^{n} \delta_{t}^{2}-\sum_{\substack{i<j, k<\ell \\
(i, j) \neq(k, \ell)}} w^{2} \frac{\left(x_{i j}, x_{k \ell}\right)}{x_{i j} x_{k \ell}}-\sum_{j<i}\left(w^{2}+w\right) \frac{\left(x_{i j}, x_{i j}\right)}{x_{i j}^{2}}\right) \delta^{-w} \\
& =\left(\Delta_{\mathfrak{h}}-\frac{1}{2}\left(w^{2}+w\right) \sum_{\alpha \in R} \frac{(\alpha, \alpha)}{\alpha^{2}}\right) \delta^{-w} .
\end{aligned}
$$

Here the middle term of the second-to-last line disappears since if we write the sum over the common denominator $\delta$ then the numerator becomes a sign semi-invariant element in $\mathbb{C}[\mathfrak{h}]$ of degree $\operatorname{deg}(\delta)-2$, and so is zero. We deduce that $L_{w}=\Theta_{w}^{\mathrm{spher}}\left(\sum_{t=1}^{n} D_{w}\left(y_{t}\right)^{2} e\right)$, as claimed.

For Cherednik algebras of type $A$ one has $(\alpha, \alpha)=2$ and an easy computation shows that $\delta \Delta_{\mathfrak{h}} \delta^{-1}=\Delta_{\mathfrak{h}}-\sum_{\alpha \in R} \frac{\delta}{\alpha} \frac{\partial}{\partial h_{\alpha}} \delta^{-1}$. Combined with (8.2.7) this shows that

$$
\delta^{w+1} \operatorname{Rad}\left(\Delta_{\mathfrak{g}}\right) \delta^{-(w+1)}=L_{w}=L_{-(w+1)} \in \Theta_{-(w+1)}^{\text {spher }}\left(\mathbf{H}_{-(w+1)}\right) .
$$

Since $\operatorname{Rad}\left(\Delta_{g}\right)$ is clearly $W$-invariant we have therefore proved that

$$
\operatorname{Rad}\left(\Delta_{\mathfrak{g}}\right) \in \mathrm{H}_{-(w+1)}^{W}=\mathrm{U}_{-(w+1)}
$$

8.4. To calculate (part of) the kernel of Rad we just have to see what happens to the element $\tau(\mathbf{1}) \in \mathscr{D}(\mathfrak{G})$. As was observed in Section 2.7, $\tau(\mathbf{1})=-\sum_{i=1}^{n} e_{i} \frac{\partial}{\partial e_{i}}$, where $\left\{e_{i}\right\}$ is a basis of $V^{*} \subset \mathbb{C}[V]$. It therefore follows from (8.2.5) that $\tau(\mathbf{1}) \cdot \mathbf{s}^{w}=-n w \mathbf{s}^{w}$. As before, $\tau(\mathbf{1}) \rho^{*}(f)=0$ for $f \in \mathbb{C}\left[\mathfrak{h}^{\text {reg }} / W\right]$ and so $\tau(\mathbf{1}) \cdot\left(\mathbf{s}^{w} \rho^{*}(f)\right)=-n w\left(\mathbf{s}^{w} \rho^{*}(f)\right.$. Thus

$$
\operatorname{Ker}(\operatorname{Rad}) \supseteq\left(\mathscr{D}(\mathfrak{G}) \cdot \tau\left(\mathfrak{s l}_{n}\right)\right)^{G}+\mathscr{D}(\mathfrak{G})^{G}(\tau(\mathbf{1})+w \operatorname{Tr}(\mathbf{1})) .
$$

The right hand side of $(\underline{8.4 .1})$ is the ideal $\left(\mathscr{D}(\mathfrak{G}) I_{-w}\right)^{G}$ in the notation of Section 2.2 and so $\mathfrak{R}$ does at least induce a homomorphism $\overline{\mathfrak{R}}: \mathfrak{D}_{-w}^{G} \rightarrow \mathrm{U}^{\text {reg }}$. 
8.5. Proof of Theorem 8.1. The proof now follows a well-worn path similar to that of [EG, Theorem 4.8 and Proposition 4.9] or [BFG, Proposition 5.4.4]. Set $c=-w$ and $\mathfrak{R}=$ $\mathfrak{R}_{c}$. Then (8.3.1) shows that $\mathfrak{R}\left(\Delta_{\mathfrak{g}}\right) \in \mathrm{U}_{c-1}$. By the proof of [BFG, Proposition 5.4.4] this is enough to ensure that $\operatorname{Im} \Re=\bigcup_{c-1}$. In fact that proof shows rather more: as $\mathfrak{R}$ is defined by restriction of differential operators it is certainly a filtered morphism and the proof of surjectivity in [BFG] is obtained by proving that the associated graded map gr $\Re$ is surjective. Therefore, $\mathfrak{R}$ is filtered surjective and hence, by (8.4.1), so is $\bar{\Re}$.

It remains to prove that $\bar{\Re}$ and gr $\bar{\Re}$ are injective. However, just as in the proof of Lemma 4.4, $\operatorname{gr} \mathfrak{D}_{c}^{G} \cong\left(\operatorname{gr}\left(\mathscr{D}(\mathfrak{G}) / \mathscr{D}(\mathfrak{G}) I_{c}\right)\right)^{G}$ is a homomorphic image of

$$
\left[\frac{\operatorname{gr} \mathscr{D}(\mathfrak{G})}{\operatorname{gr} \mathscr{D}(\mathfrak{G}) \cdot \operatorname{gr} \tau\left(I_{c}\right)}\right]^{G}=\left[\frac{\mathbb{C}\left[T^{*} \mathfrak{G}\right]}{\mathbb{C}\left[T^{*} \mathfrak{G}\right] \cdot \mu_{\mathfrak{G}}^{*}(\mathfrak{g})}\right]^{G}=\mathbb{C}[\mathcal{M}]^{G}=\mathbb{C}\left[\mathfrak{h} \times \mathfrak{h}^{*}\right]^{W},
$$

where $\mathcal{M}$ is defined in (4.3.1) and the final equality is [GG, Lemma 2.11]. In other words, $\operatorname{gr} \bar{\Re}$ is a surjective morphism from a factor of $\mathbb{C}\left[\mathfrak{h} \times \mathfrak{h}^{*}\right]^{W}$ to gr $\bigcup_{c-1}=\mathbb{C}\left[\mathfrak{h} \times \mathfrak{h}^{*}\right]^{W}$. Thus it must be an isomorphism. This also forces $\bar{\Re}$ to be an isomorphism.

\section{REFERENCES}

[BEG2] Y. Berest, P. Etingof and V. Ginzburg, Finite dimensional representations of rational Cherednik algebras, Int. Math. Res. Notes, (2003)(19), 1053-1088.

[BE] R. Bezrukavnikov and P. Etingof, Induction and restriction functors for rational Cherednik algebras. Preprint, arxiv 2008 (to appear).

[BFG] R. Bezrukavnikov, M. Finkelberg and V. Ginzburg, Cherednik algebras and Hilbert schemes in characteristic $p$, Repr. Theory 10 (2006), 254-298.

[Bj] J. E. Bjork, Filtered Noetherian rings, iㅡ Noetherian Rings and their Applications, Ed. L. W. Small, Math. Surveys and Monographs, 24, Amer. Math. Soc. Providence, RI, 1987.

[BjE] J. E. Björk and E .K. Ekström, Filtered Auslander-Gorenstein rings, in Operator algebras, unitary representations, enveloping algebras, and invariant theory. Colloque en l'honneur de J. Dixmier, Eds. A. Connes et al, Birkhauser, 1990.

[BS] M.P. Brodmann and R.Y. Sharp, Local Cohomology. An algebraic introduction with geometric applications, Cambridge University Press, Cambridge, 1998.

[De] C. Dezélée, Représentations de dimension finie de l'algèbre de Cherednik rationnelle, Bull. Soc. Math. France, 131 (2003), 465-482.

[EG] P. Etingof and V. Ginzburg, Symplectic reflection algebras, Calogero-Moser space, and deformed Harish-Chandra homomorphism, Invent. Math. 147 (2002), no. 2, 243-348.

[FG] M. Finkelberg and V. Ginzburg, Cherednik algebras for algebraic curves, arXiv:0704.3494

[GG] W. L. Gan and V. Ginzburg, Almost-commuting variety, $\mathscr{D}$-modules, and Cherednik algebras, Int. Math. Research Papers (2006), 1-54.

[GS1] I. Gordon and J. T. Stafford, Rational Cherednik algebras and Hilbert schemes. Adv. Math. 198 (2005), 222-274.

[GS2] I. Gordon and J. T. Stafford, Rational Cherednik algebras and Hilbert schemes, II: Representations and sheaves, Duke Math. J. 132 (2006), 73-135.

[Ha1] M. Haiman, Hilbert schemes, polygraphs and the Macdonald positivity conjecture, J. Amer. Math. Soc., 14 (2001) 941-1006. 
[Ha2] M. Haiman, $t, q$-Catalan numbers and the Hilbert scheme. Selected papers in honor of Adriano Garsia (Taormina, 1994). Discrete Math. 193 (1998), 201-224.

[He] G. J. Heckman, A remark on the Dunkl differential-difference operators, in Harmonic analysis on reductive groups, Prog. in Math., 101, Birkhäuser Boston, Boston, (1991), 181-191.

[Ho] M. P. Holland, Quantization of the Marsden-Weinstein reduction for extended Dynkin quivers, Ann. Sci. École Norm. Sup., 32 (1999), 813-834.

[KL] G. Krause and T. Lenagan, Growth of algebras and Gelfand-Kirillov dimension, Revised edition, Graduate Studies in Math. 22, Amer. Math. Soc., Providence, RI, 2000.

[KR] M. Kashiwara and R. Rouquier, Microlocalization of rational Cherednik algebras, to appear Duke Math. J., arXiv:0705.1245.

[Le] T. Levasseur, Relèvements d'opérateurs différentiels sur les anneaux d'invariants, in Operator Algebras, Unitary Representations, Enveloping Algebras and Invariant Theory, Eds: A. Connes et al, Prog. in Math. 92, Birkhäuser, Basel, (1990).

[MR] J. C. McConnell and J. C. Robson, Noncommutative Noetherian Rings, Grad. Texts in Math. 30, Amer. Math. Soc., Providence, RI, 2000.

$[\mathrm{Mu}]$ S. Mukai, An introduction to invariants and moduli, Cambridge Studies in Advanced Mathematics, 81. Cambridge University Press, Cambridge, 2003.

[Na] H. Nakajima, Lectures on Hilbert schemes of points on surfaces, University Lecture Series, 18, Amer. Math. Soc., Providence, RI, 1999.

[SGA1] A. Grothendieck, Revêtements étales et groupe fondamental (SGA1), IHES, Paris, 1963.

[St] J. T. Stafford, Homological properties of the enveloping algebra $U\left(\mathrm{Sl}_{2}\right)$, Math. Proc. Cambridge Philos. Soc., 91 (1982), 29-37.

[Wa] K. Watanabe, Certain invariant subrings are Gorenstein. I. Osaka J. Math. 11 (1974), 18.

(VG) Department of Mathematics, University of Chicago, Chicago, IL 60637, USA.

E-mail address: ginzburg@math.uchicago.edu

(IG) School of Mathematics and Maxwell Institute for Mathematical Sciences, EdinBurgh University, Edinburgh EH9 3JZ, Scotland.

E-mail address: i.gordon@ed.ac.uk

(JTS) Department of Mathematics, 530 Church Street, University of Michigan, Ann ArBor, MI 48109-1043, USA and Department of Mathematics, The University of Manchester, MANCHESTER, M13 9PL, UK.

E-mail address: jts@umich.edu, Toby.Stafford@manchester.ac.uk 